Pacific

Journal of

Mathematics

\title{
ON THE DEGREES OF MATRIX COEFFICIENTS OF INTERTWINING OPERATORS
}

\author{
TOBIAS FINIS, EREZ LAPID AND WERNER MÜLLER
}




\title{
ON THE DEGREES OF MATRIX COEFFICIENTS OF INTERTWINING OPERATORS
}

\author{
TOBIAS FINIS, EREZ LAPID AND WERnER MÜLleR
}

To the memory of Jonathan Rogawski

\begin{abstract}
We state and discuss a general conjectural bound on the degrees of matrix coefficients of intertwining operators for reductive groups over $p$-adic fields and a supplementary uniformity conjecture for reductive groups over number fields. We prove both conjectures for the groups GL $(r)$ and obtain partial results for other groups.
\end{abstract}

1. Introduction

2. The setup 436

3. Variants of the conjectures 438

4. Matrix coefficients of supercuspidal representations 440

5. A class of parabolic subgroups 443

6. Matrix coefficients of intertwining operators 445

7. Parabolic subgroups with abelian unipotent radical 448

References

\section{Introduction}

Let $\boldsymbol{G}$ be a reductive algebraic group defined over a $p$-adic field $F$ with residue field $\mathbb{F}_{q}$ and $G=\boldsymbol{G}(F)$. Fix a special maximal compact subgroup $K_{0}$ of $G$. For a maximal parabolic subgroup $\boldsymbol{P}=\boldsymbol{M U}$ of $\boldsymbol{G}$ and a smooth irreducible representation $\pi$ of $M=\boldsymbol{M}(F)$, we consider the family of induced representations $I_{P}(\pi, s)$, $s \in \mathbb{C}$, which extend the fixed $K_{0}$-representation $I_{P \cap K_{0}}^{K_{0}}\left(\left.\pi\right|_{M \cap K_{0}}\right)$, and the associated intertwining operators $M(s)=M_{\bar{P} \mid P}(\pi, s): I_{P}(\pi, s) \rightarrow I_{\bar{P}}(\pi,-s)$. For any open subgroup $K$ of $K_{0}$, the restriction

$$
M(s)^{K}: I_{P \cap K_{0}}^{K_{0}}\left(\left.\pi\right|_{M \cap K_{0}}\right)^{K}=I_{P}(\pi, s)^{K} \rightarrow I_{\bar{P}}(\pi,-s)^{K}=I_{\bar{P} \cap K_{0}}^{K_{0}}\left(\left.\pi\right|_{M \cap K_{0}}\right)^{K}
$$

of $M(s)$ to the space of $K$-fixed vectors is a family of linear maps between finitedimensional vector spaces which do not depend on $s$. It is well known that the

The authors were partially sponsored by grant 964-107.6/2007 from the German-Israeli Foundation for Scientific Research and Development. Finis was supported by DFG Heisenberg grant FI 1795/1-1. MSC2010: primary 20G25, 20G30; secondary 11F85.

Keywords: $p$-adic reductive groups, intertwining operators. 
matrix coefficients of the linear operators $M(s)^{K}$ are rational functions of $q^{-s}$, whose denominators can be controlled explicitly (see, e.g., [Waldspurger 2003, IV.1.1, IV.1.2]). In particular, their degrees are bounded independently of $K$ and $\pi$.

What can be said about the degrees of the numerators? In this note, we propose the following conjecture, which should provide a bound of the correct order of magnitude. Let $\boldsymbol{G}^{\prime}$ be the derived group of $\boldsymbol{G}$ and set $G^{\prime}=\boldsymbol{G}^{\prime}(F)$. Note that $K_{0}^{\prime}=K_{0} \cap G^{\prime}$ is a special maximal compact subgroup of $G^{\prime}$.

Conjecture 1. There exist constants $c>0$ and $d$, depending only on $\boldsymbol{G}$, such that for any open subgroup $K \subset K_{0}$, the degrees of the numerators of the matrix coefficients of $M(s)^{K}$ are bounded by $c \log _{q}\left[K_{0}^{\prime}: K^{\prime}\right]+d$, where $K^{\prime}=K \cap G^{\prime}$.

We also propose the following supplement in a global situation, where we consider a reductive group $\boldsymbol{G}$ defined over a number field $k$ and its base change to $F=k_{v}$ for all nonarchimedean places $v$ of $k$. Let $K_{0, v}$ be a special maximal compact subgroup of $\boldsymbol{G}\left(k_{v}\right)$.

Conjecture 2. In the global situation, assume $K_{0, v}$ to be hyperspecial for almost all places $v$ of $k$. Then Conjecture 1 is true for all pairs of local groups $\boldsymbol{G}\left(k_{v}\right)$ and $K_{0, v}$, with uniform values of $c$ and $d$.

It is equivalent to consider the normalized intertwining operators $R(s)$ defined by Arthur [1989]. We discuss this modification and some other simple variants in Section 3 below.

The main result of this paper is the following.

Theorem 1. Conjectures 1 and 2 are true for the groups $\boldsymbol{G}=\mathrm{GL}(r)$. More precisely, the constants $c$ and $d$ in Conjecture 1 depend only on $r$ and $\left[F: \mathbb{Q}_{p}\right]$.

An important motivation for our paper is provided by the analysis of limit multiplicities for noncompact quotients of $\boldsymbol{G}(\mathbb{R})$, where in order to deal with the spectral side of Arthur's trace formula, it is crucial to bound the degrees of the matrix coefficients of local intertwining operators. This application (for $\boldsymbol{G}=\mathrm{GL}(r)$ ) is discussed in [Finis et al. 2012]. We opted to single out our conjectures and results on local intertwining operators as a separate paper, since they may be of interest in their own right.

A natural analog of Conjecture 1 in the archimedean case $(F=\mathbb{R}$ or $\mathbb{C})$ has been obtained in [Lapid 2004]. To explain it, fix a maximal compact subgroup $K_{0}$ of $G$ (it is well known to be unique up to conjugation). For any $K_{0}$-module $V$ and $\sigma \in \widehat{K}_{0}$, let $V^{\sigma}$ denote the $\sigma$-isotypic part of $V$. Let $R(\pi, s): I_{P}(\pi, s) \rightarrow I_{\bar{P}}(\pi,-s)$ be the normalized intertwining operators and $R(\pi, s)^{\sigma}$ their restrictions to linear maps between the finite-dimensional vector spaces $I_{P}(\pi, s)^{\sigma}$ and $I_{\bar{P}}(\pi,-s)^{\sigma}$ which do not depend on $s$. The matrix coefficients of the operators $R(\pi, s)$ are rational functions of $s$ [Arthur 1989, Theorem 2.1]. We denote by $\|\sigma\|$ the maximum of 
the norms of the highest weights of $\sigma$ (with respect to a fixed choice of norm on the vector space spanned by the lattice of characters of a maximal torus of the connected component of the identity of $K_{0}$ ). Then we can formulate the following direct consequence of [Lapid 2004, Proposition A.2].

Theorem 2. There exists a constant $c>0$, depending only on $\boldsymbol{G}$ and the norm $\|\cdot\|$, such that for any maximal parabolic subgroup $\boldsymbol{P}=\boldsymbol{M U}$ of $\boldsymbol{G}$, any irreducible representation $\pi$ of $M$, and any $K_{0}$-type $\sigma \in \widehat{K}_{0}$, the degrees of the matrix coefficients of $R(\pi, s)^{\sigma}$ are bounded by $c\|\sigma\|$.

Let us now make a few comments about the proof of Theorem 1, at the same time outlining the partial results that we can prove for general groups $\boldsymbol{G}$. By a standard argument, we can reduce to the case where $\pi$ is supercuspidal. Furthermore, a result of Lubotzky (quoted as Proposition 3 below) allows us to assume that $K^{\prime}$ is a principal congruence subgroup of $G^{\prime}$. After these preliminary reductions, there are two main ingredients. First, assuming the widely believed conjecture that supercuspidal representations of $G$ are induced from open subgroups which are compact modulo the center, ${ }^{1}$ we can deduce a good bound for the support of matrix coefficients of these representations (property (PSC) of Definition 7 below). This inference is an explication of an argument which goes back to [Jacquet 1971] (cf. [Bushnell 1990]). The classification of supercuspidals needed for our argument has been proven for $\boldsymbol{G}=\mathrm{GL}(r)$ by Bushnell and Kutzko [1993a]. It is also known in many other cases, most notably for classical groups of odd residual characteristic [Stevens 2008] and for any group in large residual characteristic [Kim 2007]. Therefore, property (PSC) is true in these cases.

The second part of the main argument is a simple proof of the rationality of intertwining operators for parabolic subgroups $\boldsymbol{P}$ with abelian unipotent radical, ${ }^{2}$ which allows us to control the degrees of the rational functions involved (Proposition 16 and Theorem 21). For $\boldsymbol{G}=\mathrm{GL}(r)$, this fortunately covers all cases, thereby completing the proof of Theorem 1. The technical geometric property that is needed for our argument is explicated in Definition 15 below. It is unfortunately not satisfied for all maximal parabolic subgroups, even in the case of classical groups (see Remark 18). It is conceivable that a more elaborate argument will work in general.

Acknowledgements. We are grateful to Joseph Bernstein, Colin Bushnell, Guy Henniart and Eitan Sayag for useful discussions. We thank the Centre Interfacultaire Bernoulli, Lausanne, and the Max Planck Institute for Mathematics, Bonn, where a part of this paper was worked out.

\footnotetext{
${ }^{1}$ In fact, it suffices to assume that every supercuspidal representation is contained in such an induced representation of finite length (see Section 4 below for more details).

${ }^{2}$ We also make the additional technical assumption that the group $\boldsymbol{G}$ is split over $F$.
} 


\section{The setup}

Let $F$ be a $p$-adic field with normalized absolute value $|\cdot|$, ring of integers $\mathbb{O}$, and uniformizer $\varpi$. Let $q$ be the cardinality of the residue field of $F$.

As a rule, we write $X=\boldsymbol{X}(F)$ whenever $\boldsymbol{X}$ is a variety over $F$. Let $\boldsymbol{G}$ be a connected reductive algebraic group defined over $F$ with center $\boldsymbol{Z}$. All algebraic subgroups that will be considered in the sequel are implicitly assumed to be defined over $F$. Let $\boldsymbol{G}^{\prime}$ be the derived group of $\boldsymbol{G}$ and for any subgroup $K \subset G$, write $K^{\prime}=K \cap G^{\prime}$. Fix a maximal $F$-split torus $\boldsymbol{T}_{0}$ and a minimal parabolic subgroup $\boldsymbol{P}_{0}=\boldsymbol{M}_{0} \boldsymbol{U}_{0} \supset \boldsymbol{T}_{0}$ of $\boldsymbol{G}$, where $\boldsymbol{M}_{0}=C_{\boldsymbol{G}}\left(\boldsymbol{T}_{0}\right)$ is a minimal Levi subgroup of $\boldsymbol{G}$. Let $\Phi=R\left(\boldsymbol{T}_{0}, \boldsymbol{G}\right)$ be the set of roots of $\boldsymbol{T}_{0}$. The choice of $\boldsymbol{P}_{0}$ fixes a set of positive roots $R\left(\boldsymbol{T}_{0}, \boldsymbol{U}_{0}\right) \subset \Phi$. Let $\Delta_{0} \subset \Phi$ be the corresponding subset of simple roots. The standard maximal parabolic subgroups of $\boldsymbol{G}$ correspond bijectively to the simple roots, and for $\alpha \in \Delta_{0}$, we denote by $\boldsymbol{P}^{\alpha}=\boldsymbol{M}^{\alpha} \boldsymbol{U}^{\alpha}$ the unique standard maximal parabolic subgroup with $\alpha \in R\left(\boldsymbol{T}_{0}, \boldsymbol{U}^{\alpha}\right)$. For any Levi subgroup $\boldsymbol{M}$, we denote by $\mathscr{P}(\boldsymbol{M})$ the (finite) set of all parabolic subgroups of $\boldsymbol{G}$ with Levi part $\boldsymbol{M}$. For any standard parabolic subgroup $\boldsymbol{P}$ of $\boldsymbol{G}$ with standard Levi decomposition $\boldsymbol{P}=\boldsymbol{M} \boldsymbol{U}$, we denote by $\overline{\boldsymbol{P}}=\boldsymbol{M} \overline{\boldsymbol{U}}$ the opposite parabolic subgroup.

Fix a special maximal compact subgroup $K_{0}$ of $G$ (more precisely, the stabilizer of a special point in the apartment associated to $\boldsymbol{T}_{0}$ ), so that we have the Iwasawa decomposition $P_{0} K_{0}=G$. In addition, we have the Cartan decomposition $G=$ $K_{0} M_{0}^{+} K_{0}$, where $M_{0}^{+}$is the set of all $m \in M_{0}$ with $|\alpha(m)| \geq 1$ for all $\alpha \in \Delta_{0}$ [Tits 1979, §3.3]. Also, for any parabolic subgroup $\boldsymbol{P}=\boldsymbol{M U}$ with Levi subgroup $\boldsymbol{M} \supset \boldsymbol{M}_{0}$, we have $\left(P \cap K_{0}\right)=\left(M \cap K_{0}\right)\left(U \cap K_{0}\right)$. We take a representative $w_{0} \in K_{0}$ for the longest Weyl element. Fix a faithful representation $\rho: \boldsymbol{G} \rightarrow \operatorname{GL}(V)$ and an O-lattice $\Lambda_{V}$ in the representation space $V$ such that $K_{0}=\left\{g \in G: \rho(g) \Lambda_{V}=\Lambda_{V}\right\}$, and for $n=1,2, \ldots$, let

$$
K_{n}=\left\{g \in G: \rho(g) v \equiv v\left(\bmod \varpi^{n} \Lambda_{V}\right), v \in \Lambda_{V}\right\}
$$

be the associated principal congruence subgroups of $K_{0}$. Note that a more natural filtration of $K_{0}$ has been defined in terms of the Bruhat-Tits building of $G^{\prime}$ in [Schneider and Stuhler 1997, Chapter I].

Suppose now that $\boldsymbol{P}=\boldsymbol{M U}$ is a standard maximal parabolic subgroup. Let $\chi_{P}$ be the fundamental weight of $\boldsymbol{P}$. Some integral power of $\chi_{P}$ defines a rational character of $\boldsymbol{P}$ trivial on $\boldsymbol{U}$. Therefore $\left|\chi_{P}\right|$ defines a character $\left|\chi_{P}\right|: P \rightarrow \mathbb{R}_{>0}$ and we can extend this character uniquely to a right- $K_{0}$-invariant function, still denoted by $\left|\chi_{P}\right|$, on $G$. Let $\left(\pi, V_{\pi}\right)$ be an irreducible (smooth) representation of $M$. Let $\delta_{P}$ be the modulus function of $P$. Consider the family of induced representations $I_{P}(\pi, s), s \in \mathbb{C}$, of $G$ which extend the $K_{0}$-representation $I_{P \cap K_{0}}^{K_{0}}\left(\left.\pi\right|_{M \cap K_{0}}\right)$. Namely, $I_{P}(\pi, s)$ is the space of all smooth functions $\varphi: G \rightarrow V_{\pi}$ with 


$$
\varphi(p g)=\left|\chi_{P}\right|(p)^{s} \delta_{P}(p)^{1 / 2} \pi(p) \varphi(g)
$$

for all $p \in P, g \in G$, where $\pi$ is extended to $P$ via the canonical projection $P \rightarrow M$, and the $G$-action is given by right translations. Any smooth function $\varphi: K_{0} \rightarrow V_{\pi}$ with $\varphi(p k)=\pi(p) \varphi(k)$ for all $k \in P \cap K_{0}$ extends uniquely to a function $\varphi_{s} \in I_{P}(\pi, s)$. Let $\pi^{\vee}$ be the contragredient of $\pi$ and denote the pairing between $V_{\pi}$ and $V_{\pi} \vee$ by $(\cdot, \cdot)$. Then

$$
\left(\varphi, \varphi^{\vee}\right)=\int_{K_{0}}\left(\varphi(k), \varphi^{\vee}(k)\right) d k
$$

defines a pairing between $I_{P}(\pi, s)$ and $I_{P}\left(\pi^{\vee},-s\right)$. Fix a choice of Haar measure on $\bar{U}$. The intertwining operators $M(s)=M_{\bar{P} \mid P}(\pi,-s): I_{P}(\pi, s) \rightarrow I_{\bar{P}}(\pi, s){ }^{3}$ which are defined by the meromorphic continuation of the integrals

$$
(M(s) \varphi)(g)=\int_{\bar{U}} \varphi(\bar{u} g) d \bar{u}, \quad \varphi \in I_{P}(\pi, s),
$$

were first studied in this generality by Harish-Chandra. (See [Waldspurger 2003, Section IV] for a self-contained treatment.) It is known that the matrix coefficients $\left(M(s) \varphi_{s}, \varphi_{s}^{\vee}\right)$ for $\varphi \in I_{P \cap K_{0}}^{K_{0}}\left(\left.\pi\right|_{M \cap K_{0}}\right)$ and $\varphi^{\vee} \in I_{\bar{P} \cap K_{0}}^{K_{0}}\left(\left.\pi^{\vee}\right|_{M \cap K_{0}}\right)$ are rational functions of $q^{-s}$ [Waldspurger 2003, IV.1.1] and that the degree of the denominator is bounded in terms of $\boldsymbol{G}$ only [Waldspurger 2003, IV.1.2]; see also [Shahidi 1981, Theorems 2.2.1, 2.2.2; Silberger 1979]. It is often advantageous to work instead with the normalized intertwining operators $R(s)=R_{\bar{P} \mid P}(\pi, s): I_{P}(s) \rightarrow I_{\bar{P}}(-s)$ defined in [Arthur 1989], which differ from $M(s)$ by a certain rational function of $q^{-s}$ depending on $\pi$ whose degree is bounded in terms of $G$ only. Thus, the matrix coefficients of $R(s)$ are also rational functions in $q^{-s}$ and the degree of the denominator is bounded in terms of $G$.

Occasionally we will also consider intertwining operators for general (nonmaximal) parabolic subgroups containing $\boldsymbol{T}_{0}$. For this, let $\boldsymbol{M} \supset \boldsymbol{M}_{0}$ be a Levi subgroup of $\boldsymbol{G}$ and set $\mathfrak{a}_{M, \mathbb{C}}^{*}=X^{*}(\boldsymbol{M}) \otimes \mathbb{C}$, where $X^{*}(\boldsymbol{M})$ denotes the group of ( $F$-rational) characters of $\boldsymbol{M}$. Then for any smooth irreducible representation $\pi$ of $M$, we have the families of induced representations $I_{P}(\pi, \lambda), \boldsymbol{P} \in \mathscr{P}(\boldsymbol{M}), \lambda \in \mathfrak{a}_{M, \mathbb{C}}^{*}$, and the associated intertwining operators $M_{P_{2} \mid P_{1}}(\pi, \lambda): I_{P_{1}}(\pi, \lambda) \rightarrow I_{P_{2}}(\pi, \lambda)$ for pairs of parabolic subgroups $\boldsymbol{P}_{1}, \boldsymbol{P}_{2} \in \mathscr{P}(\boldsymbol{M})$ [Waldspurger 2003, p. 278]. We can extend arbitrary functions $\varphi \in I_{P_{1} \cap K_{0}}^{K_{0}}\left(\left.\pi\right|_{M \cap K_{0}}\right)$ and $\varphi^{\vee} \in I_{P_{2} \cap K_{0}}^{K_{0}}\left(\left.\pi^{\vee}\right|_{M \cap K_{0}}\right)$ uniquely to functions $\varphi_{\lambda} \in I_{P_{1}}(\pi, \lambda)$ and $\varphi_{-\lambda}^{\vee} \in I_{P_{2}}\left(\pi^{\vee},-\lambda\right)$, respectively, and the matrix coefficients $\left(M(\lambda) \varphi_{\lambda}, \varphi_{-\lambda}^{\vee}\right)$ are rational functions of the variables $q^{-\left\langle\lambda, \alpha^{\vee}\right\rangle}, \alpha \in \Delta_{\boldsymbol{P}}$. Here $\Delta_{\boldsymbol{P}}$ is the set of simple roots of $\boldsymbol{U}$. The degree of the denominator is bounded in terms of $\boldsymbol{G}$ only. The normalized intertwining operator $R_{P_{2} \mid P_{1}}(\pi, \lambda)$ differs from

\footnotetext{
${ }^{3}$ Note that $I_{\bar{P}}(\pi,-s)$ is defined using $\chi_{\bar{P}}$ and $\delta_{\bar{P}}$ and that $\left.\chi_{\bar{P}}\right|_{M}=\left.\chi_{P}^{-1}\right|_{M}$ and $\left.\delta_{\bar{P}}\right|_{M}=\left.\delta_{P}^{-1}\right|_{M}$.
} 
the operator $M_{P_{2} \mid P_{1}}(\pi, \lambda)$ by a normalizing scalar which is a rational function of $q^{-\left\langle\lambda, \alpha^{\vee}\right\rangle}$ of degree bounded in terms of $\boldsymbol{G}$ only.

Let $\mathfrak{g}=$ Lie $\boldsymbol{G}$ and denote by Ad : $\boldsymbol{G} \rightarrow \mathrm{GL}(\mathfrak{g})$ the adjoint representation. Fix an O-lattice $\Lambda \subset \mathfrak{g}$ stabilized by the operators $\operatorname{Ad}(k), k \in K_{0}$, and define a norm on $\mathfrak{g}$ by $\left\|\sum_{i=1}^{d} t_{i} X_{i}\right\|_{\mathfrak{g}}=\max _{1 \leq i \leq d}\left|t_{i}\right|$ for an (arbitrary) 0 -basis $X_{1}, \ldots, X_{d}$ of $\Lambda$. This defines a norm $\|\cdot\|_{\operatorname{End}(\mathfrak{g})}$ on $\operatorname{End}(\mathfrak{g})$; namely, $\|A\|_{\operatorname{End}(\mathfrak{g})}$ is the maximum of the absolute values of the matrix coefficients of $A$ with respect to the basis $X_{1}, \ldots, X_{d}$. For any $g \in G$, we write $\|g\|_{G}=\|\operatorname{Ad}(g)\|_{\operatorname{End}(\mathfrak{g})}$, and for any real number $R$ we set $\mathscr{B}^{G}(R)=\left\{g \in G:\|g\|_{G} \leq q^{R}\right\}$, which is a compact set modulo $Z$. We often omit the index $G$ from $\|\cdot\|_{G}$ and $\mathscr{B}^{G}(R)$ if it is clear from the context.

In the global situation of a reductive group $\boldsymbol{G}$ defined over a number field $k$, we need of course to fix analogous global data that induce the local data pertaining to $\boldsymbol{G}\left(k_{v}\right)$ for the nonarchimedean places $v$ of $k$. In particular, we fix an $\mathbb{O}_{k}$-lattice $\Lambda \subset \mathfrak{g}$ to define the local norms $\|\cdot\|_{\boldsymbol{G}\left(k_{v}\right)}$ via base change to $\mathcal{O}_{k_{v}}$. In the same way, we obtain the representation $\rho_{v}$ and the lattice $\Lambda_{V_{v}} \subset V_{v}$ intervening in the definition of the groups $K_{n, v}$ from a representation $\rho: \boldsymbol{G} \rightarrow \mathrm{GL}(V)$ defined over $k$ and an $\mathcal{O}_{k}$-lattice $\Lambda_{V}$ in the $k$-vector space $V$. It is well known that $K_{0, v}$ is then hyperspecial for almost all $v$.

We write $A \ll B$ (or $B \gg A$ ) if there exists a constant $c$ (independent of other quantities) such that $A \leq c B$.

\section{Variants of the conjectures}

In this section we discuss some simple variants of Conjectures 1 and 2. In studying our conjectures, it is useful to restrict attention to the principal congruence subgroups $K_{n}^{\prime}$ of $K_{0}^{\prime}$. This is possible by the following statement, which is a special case of [Lubotzky 1995, Lemma 1.6].

Proposition 3 (Lubotzky). There exist constants $c_{0}$ and $d_{0}$ such that any open subgroup $K$ of $K_{0}$ contains the principal congruence subgroup $K_{n}^{\prime}$ of $G^{\prime}$ for $n=$ $\left\lfloor c_{0} \log _{q}\left[K_{0}^{\prime}: K^{\prime}\right]+d_{0}\right\rfloor$. Moreover, if $\boldsymbol{G}$ is defined over a number field $k$ and for any finite place $v, K_{0, v}$ is a special maximal compact subgroup of $\boldsymbol{G}\left(k_{v}\right)$, which is hyperspecial for almost all $v$, then for the pairs $\left(\boldsymbol{G}\left(k_{v}\right), K_{0, v}\right)$, one may take uniform values of $c_{0}$ and $d_{0}$ (in fact, $c_{0}=\left[k_{v}: \mathbb{Q}_{p}\right]$ works for almost all $v$ ).

Remark 4. Note that in [Lubotzky 1995] it is assumed that $\boldsymbol{G}^{\prime}$ is simply connected, and one can then take $d_{0}=0$. The general case follows easily by passing to the simply connected covering group of $\boldsymbol{G}^{\prime}$.

Proposition 3 implies that equivalent forms of Conjectures 1 and 2 are obtained by replacing the index $\left[K_{0}^{\prime}: K^{\prime}\right]$ by the level of $K^{\prime}$, which is defined as

$$
\operatorname{level}\left(K^{\prime}\right):=q^{n},
$$


where $n \geq 0$ is the smallest integer with $K^{\prime} \supset K_{n}^{\prime}$.

We now consider the generalization of our conjectures to arbitrary parabolic subgroups and the associated intertwining operators.

Proposition 5. Suppose that Conjecture 1 is true for any Levi subgroup $\boldsymbol{L} \supset \boldsymbol{M}_{0}$ in place of $\boldsymbol{G}$. Then there exist constants $c>0$ and $d$, depending only on $\boldsymbol{G}$, such that for any open subgroup $K \subset K_{0}$, the degrees of the numerators of the matrix coefficients of $M_{P_{2} \mid P_{1}}(\lambda)^{K}$, as rational functions of the variables $q^{-\left\langle\lambda, \alpha^{\vee}\right\rangle}, \alpha \in \Delta_{\boldsymbol{P}}$, are bounded by $c \log _{q}\left[K_{0}^{\prime}: K^{\prime}\right]+d$.

In the global situation of a reductive group $\boldsymbol{G}$ defined over a number field $k$, suppose that Conjecture 2 is true for all $\boldsymbol{L} \supset \boldsymbol{M}_{0}$. Then the degree bound above holds for the local groups $\boldsymbol{G}\left(k_{v}\right)$ and $K_{0, v}$ with uniform values of $c$ and $d$ as $v$ ranges over the nonarchimedean places of $k$.

Proof. Let $\boldsymbol{P}_{1}=\boldsymbol{Q}_{0}, \boldsymbol{Q}_{1}, \ldots, \boldsymbol{Q}_{l}=\boldsymbol{P}_{2}$ be a sequence of adjacent parabolic subgroups from $\boldsymbol{P}_{1}$ to $\boldsymbol{P}_{2}$ and let $\Delta \boldsymbol{Q}_{i} \cap \Delta \boldsymbol{Q}_{i+1}=\left\{\alpha_{i}\right\}$. We can decompose $M_{P_{2} \mid P_{1}}(\pi, \lambda)$ into a product of rank-one intertwining operators $M_{Q_{i+1} \mid Q_{i}}\left(\pi,\left\langle\lambda, \alpha_{i}^{\vee}\right\rangle\right)$. Thus, it is enough to consider the degrees of the matrix coefficients of $M_{Q_{i+1} \mid Q_{i}}\left(\sigma,\left\langle\lambda, \alpha_{i}^{\vee}\right\rangle\right)^{K}$, $i=0, \ldots, l-1$. Fix $i$ and let $\boldsymbol{R}=\boldsymbol{M}_{\boldsymbol{R}} \boldsymbol{N}_{\boldsymbol{R}}$ be the parabolic subgroup generated by $\boldsymbol{Q}_{i}$ and $\boldsymbol{Q}_{i+1}$. Let $\boldsymbol{Q}^{\prime}=\boldsymbol{M}_{\boldsymbol{R}} \cap \boldsymbol{Q}_{i}$ and $\boldsymbol{Q}^{\prime \prime}=\boldsymbol{M}_{\boldsymbol{R}} \cap \boldsymbol{Q}_{i+1}$. Then $\boldsymbol{Q}^{\prime}$ and $\boldsymbol{Q}^{\prime \prime}$ are maximal parabolic subgroups of $\boldsymbol{M}_{\boldsymbol{R}}$ with Levi subgroup $\boldsymbol{M}$ and $\boldsymbol{Q}^{\prime \prime}=\overline{\boldsymbol{Q}^{\prime}}$. By [Waldspurger 2003, p. 284, (14)], the matrix coefficients of $M_{Q_{i+1} \mid Q_{i}}\left(\sigma,\left\langle\lambda, \alpha_{i}^{\vee}\right\rangle\right)^{K}$ are given by those of $M_{\overline{Q^{\prime}} \mid Q^{\prime}}\left(\sigma,\left\langle\lambda, \alpha_{i}^{\vee}\right\rangle\right)^{K \cap M_{R}}$, and the degrees of the latter coefficients satisfy by assumption the bounds of Conjectures 1 and 2 .

Finally, it is clear that we can replace the intertwining operators $M(s)$ and $M(\lambda)$ by the normalized intertwining operators $R(s)$ and $R(\lambda)$ in Conjectures 1 and 2 and Proposition 5. In fact, we can obtain slightly stronger statements for the normalized operators. If we replace $M(s)$ by $R(s)$ in Conjecture 1 , and in addition $\boldsymbol{G}$ is unramified and $K_{0}$ hyperspecial, then we may take $d=0$, since any representation which admits a $K_{0}^{\prime}$-fixed vector is a twist by a character of $G / G^{\prime}$ of an unramified representation of $G$. Similarly, by Remark 4, we may take $d=0$ in the analog of Conjecture 2 for $R(s)$, if $\boldsymbol{G}^{\prime}$ is simply connected and we omit the finitely many places $v$ where $\boldsymbol{G}\left(k_{v}\right)$ is ramified or $K_{0, v}$ not hyperspecial. The same remarks apply to Proposition 5. If we consider here level $\left(K^{\prime}\right)$ instead of $\left[K_{0}^{\prime}: K^{\prime}\right]$, then we do not need to make any additional assumption on $G^{\prime}$, since trivially $\log _{q} \operatorname{level}\left(K^{\prime}\right) \geq 1$ whenever $K^{\prime} \neq K_{0}^{\prime}$. We record the resulting variant of Proposition 5 explicitly, since we intend to use the statement in another paper.

Proposition 6. Suppose that Conjecture 1 is true for any Levi subgroup $\boldsymbol{L} \supset \boldsymbol{M}_{0}$ of $\boldsymbol{G}$. Then there exists a constant $c>0$, depending only on $\boldsymbol{G}$, such that for any open subgroup $K \subset K_{0}$, the degrees of the numerators of the matrix coefficients of $R_{P_{2} \mid P_{1}}(\lambda)^{K}$, as rational functions of the variables $q^{-\left\langle\lambda, \alpha^{\vee}\right\rangle}, \alpha \in \Delta_{\boldsymbol{P}}$, are 
bounded by $c \log _{q} \operatorname{level}\left(K^{\prime}\right)$ if $\boldsymbol{G}$ is unramified and $K_{0}$ is hyperspecial, and by $c\left(\log _{q} \operatorname{level}\left(K^{\prime}\right)+1\right)$ otherwise.

In the global situation of a reductive group $\boldsymbol{G}$ defined over a number field $k$, suppose that Conjecture 2 is true for all $\boldsymbol{L} \supset \boldsymbol{M}_{0}$. Then the degree bound above for the numerators of the matrix coefficients of $R_{P_{2} \mid P_{1}}(\lambda)^{K}$ holds with a uniform value of $c$ for all local groups $\boldsymbol{G}\left(k_{v}\right)$ and $K_{0, v}$ as $v$ ranges over the nonarchimedean places of $k$.

\section{Matrix coefficients of supercuspidal representations}

Definition 7. We say that $G$ has polynomially bounded support of supercuspidal matrix coefficients (PSC) if there exist constants $c$ and $d$ such that for every open subgroup $K \subset K_{0}$ and any supercuspidal representation $\pi$ of $G$, the support of the matrix coefficients $\left(\pi(g) v, v^{\vee}\right), v \in \pi^{K}, v^{\vee} \in\left(\pi^{\vee}\right)^{K}$, is contained in $\mathscr{B}\left(c \log _{q}\left[K_{0}^{\prime}: K^{\prime}\right]+d\right)$.

Note that property (PSC) is independent of the choice of $K_{0}$, which could be replaced by an arbitrary open compact subgroup of $G$. However, the possible values of the constants $c$ and $d$ will depend on $K_{0}$ (and the norm $\|\cdot\|_{G}$ on $\mathfrak{g}$ ).

Conjecture 3. Every p-adic reductive group $G$ has property (PSC).

We will show that this conjecture is true in a large number of cases. In addition, we will obtain a global uniformity statement for the constants $c$ and $d$ for reductive groups $\boldsymbol{G}$ defined over number fields $k$ and almost all of the associated local groups $\boldsymbol{G}\left(k_{v}\right)$ (see Corollary 13 below).

Let $L$ be an open subgroup of $G$ containing $Z$ such that $L / Z$ is compact. We refer to such subgroups as open compact modulo center (ocmc) for short. We say that a finite-dimensional representation $\sigma$ of $L$ is cuspidal if for every proper parabolic subgroup $\boldsymbol{P}$ of $\boldsymbol{G}$ with unipotent radical $\boldsymbol{U}$, we have $\sigma^{L \cap U}=0$. Here, it clearly suffices to consider only maximal parabolic subgroups. By [Bushnell 1990, Theorem 1 supp.], this condition is necessary (and in fact also sufficient, by Lemma 8 below) for $\operatorname{Ind}_{L}^{G} \sigma$ to be of finite length, in which case it is the direct sum of finitely many irreducible supercuspidal representations. Note that if $\sigma$ is cuspidal, then its contragredient $\sigma^{\vee}$ is cuspidal as well. We say that a supercuspidal representation $\pi$ of $G$ is induced from an ocmc, if there exists a pair $(L, \sigma)$ where $L$ is an ocmc and $\sigma \in \hat{L}$, necessarily cuspidal, such that $\pi=\operatorname{Ind}_{L}^{G} \sigma$.

It is widely believed that every irreducible supercuspidal representation $\pi$ is induced from an ocmc, ${ }^{4}$ and in fact this is known in many cases (see [Bushnell and Kutzko 1993a; Kim 2007; Stevens 2008; Yu 2001], and earlier work by Howe,

\footnotetext{
${ }^{4}$ We were unable to trace back who precisely formulated the conjecture in this generality, but it certainly goes back to the early days of the representation theory of $p$-adic groups.
} 
Morris, Moy and others). For our purposes it suffices to know that $\pi$ is a constituent of $\operatorname{Ind}_{L}^{G} \sigma$ for some cuspidal $\sigma$.

Lemma 8. Let $L$ be an ocmc. Then there exist constants $c$, depending only on $G$, and $d$, depending on $L$, such that for any cuspidal $\sigma \in \hat{L}$, any open subgroup $K \subset K_{0}$ and any $f \in\left(\operatorname{Ind}_{L}^{G} \sigma\right)^{K}$ we have $\operatorname{supp}(f) \subset \mathscr{B}\left(c \log _{q}\left[K_{0}^{\prime}: K^{\prime}\right]+d\right)$.

Proof. Note first that the assertion is trivial if $\boldsymbol{G}^{\prime}$ is anisotropic, since $G / Z$ is then compact. So, we may assume that the $F$-rank of $\boldsymbol{G}^{\prime}$ is nonzero. By Lubotzky's result (Proposition 3 above), we may assume without loss of generality that $K^{\prime}$ is a principal congruence subgroup $K_{n}^{\prime}$ of $G^{\prime}$. In particular, $K^{\prime}$ is normal in $K_{0}$.

Let $g \in G$ and write its Cartan decomposition as $g=k_{1} a k_{2} \in G$ with $k_{1}, k_{2} \in K_{0}$ and $a \in M_{0}^{+}$. We first show that there are constants $c$ and $d$ such that $\|g\|>q^{c n+d}$ implies the existence of a standard maximal parabolic subgroup $\boldsymbol{P}=\boldsymbol{M U}$ of $\boldsymbol{G}$ satisfying

$$
U \cap k^{-1} L k \subset a(U \cap K) a^{-1} \text { for all } k \in K_{0} .
$$

Assume that $\|g\|=\|a\|>q^{c n+d}$ for some $c>0$ and $d$ which will be specified later. Note first that there are only finitely many $K_{0}$-conjugates of the group $L$, and that their intersections with $U_{0}$ generate an open compact subgroup $V_{0}(L)$ of $U_{0}$. Using the exponential map, we can identify $\boldsymbol{U}_{0}$ with its Lie algebra, which is an affine space. Fixing a norm on $U_{0}$, we let $U_{0}(n)$ be the lattice consisting of the elements of $U_{0}$ of norm bounded by $q^{n}$ and set $U(n)=U_{0}(n) \cap U$ for any standard parabolic subgroup $P=M U$ of $G$. Clearly, there exists a constant $n_{0}=n_{0}(L)$ such that $V_{0}(L)$ is contained in $U_{0}\left(n_{0}\right)$, and therefore the left-hand side of (1) is contained in $U\left(n_{0}\right)$ for all $k \in K_{0}$.

Let $\beta \in \Delta_{0}$ with $|\beta(a)|=\max _{\alpha \in \Delta_{0}}|\alpha(a)|$. There exist constants $c_{1}>0$ and $n_{1}$ such that $\max _{\alpha \in \Delta_{0} \cup-\Delta_{0}}|\alpha(b)| \geq q^{-n_{1}}\|b\|^{c_{1}}$ for any $b \in M_{0}$. Therefore, we obtain from $|\alpha(a)| \geq 1, \alpha \in \Delta_{0}$, and $\|a\|>q^{c n+d}$ that $|\beta(a)|>q^{c_{1} c n+c_{1} d-n_{1}}$, which implies in turn that $|\alpha(a)|>q^{c_{1} c n+c_{1} d-n_{1}}$ for all roots $\alpha \in R\left(\boldsymbol{T}_{0}, \boldsymbol{U}^{\beta}\right)$. There also exists a constant $n_{2}$ such that $U^{\beta} \cap K=U^{\beta} \cap K_{n}^{\prime}$ contains $U^{\beta}\left(-n-n_{2}\right)$, which implies that $a\left(U^{\beta} \cap K\right) a^{-1}$ contains $U^{\beta}\left(c_{1} c n+c_{1} d-n_{1}-n-n_{2}\right)$. It is therefore sufficient to take $c=c_{1}^{-1}$ and $d=c_{1}^{-1}\left(n_{0}+n_{1}+n_{2}\right)$ to obtain (1) for $P=P^{\beta}$.

Let now $\pi=\operatorname{Ind}_{L}^{G} \sigma$. For an arbitrary element $f \in \pi^{K}$, set $f_{2}=\pi\left(k_{2}\right) f \in \pi^{K^{\prime}}$. For any $u \in U \cap K=U \cap K^{\prime}$, we have

$$
f(g)=f_{2}\left(k_{1} a\right)=f_{2}\left(k_{1} a u\right)=f_{2}\left(u^{\prime} k_{1} a\right),
$$

where $u^{\prime}=k_{1} a u a^{-1} k_{1}^{-1}$. If in addition $u^{\prime} \in k_{1} U k_{1}^{-1} \cap L$, then we get $f(g)=$ $\sigma\left(u^{\prime}\right) f_{2}\left(k_{1} a\right)=\sigma\left(u^{\prime}\right) f(g)$. Using (1) and the cuspidality of $\sigma$, we conclude that $f(g) \in \sigma^{k_{1} U k_{1}^{-1} \cap L}=0$. 
Remark 9. The qualitative statement that in the situation of the lemma any element of $\operatorname{Ind}_{L}^{G} \sigma$ has compact support modulo the center is contained in [Bushnell 1990, Theorem 1 supp.] in the case $\boldsymbol{G}=\mathrm{GL}(r)$. The argument is originally due to Jacquet [1971].

Corollary 10. There exist constants $c^{\prime}$ and $d^{\prime}$ with the following property. Let $L$ be an ocmc of $G, \sigma$ be a cuspidal representation of $L$, and $\pi=\operatorname{Ind}_{L}^{G} \sigma$. Let $K \subset K_{0}$ be open and let $v \in \pi^{K}$ and $v^{\vee} \in\left(\pi^{\vee}\right)^{K}$. Then the support of $\left(\pi(g) v, v^{\vee}\right)$ is contained in $\mathscr{B}\left(c^{\prime} \log _{q}\left[K_{0}^{\prime}: K^{\prime}\right]+d^{\prime}\right)$.

Proof. Clearly, if $\sigma$ is a cuspidal representation of an ocmc $L_{1}$ and $L \supset L_{1}$ is a larger ocmc, then $\operatorname{Ind}_{L_{1}}^{L} \sigma$ is a cuspidal representation of $L$ [Bushnell 1990]. We can therefore assume that $L$ is a maximal ocmc. In other words, denoting by $\boldsymbol{T}_{\boldsymbol{G}}$ the maximal $F$-split torus of $\boldsymbol{Z}, L$ is the inverse image under the projection $G \rightarrow G / T_{G}$ of a maximal compact subgroup of $G / T_{G}$, which is also the group of $F$-points of the algebraic group $\boldsymbol{G} / \boldsymbol{T}_{\boldsymbol{G}}$, since the first Galois cohomology group of $\boldsymbol{T}_{\boldsymbol{G}}$ is trivial. There are finitely many such subgroups $L$ up to $G$-conjugation [Tits 1979, §3.2]. It follows from the previous lemma that for suitable positive constants $c$ and $d$, the supports $S$ and $S^{\vee}$ of $v \in \pi^{K}$ and $v^{\vee} \in\left(\pi^{\vee}\right)^{K}$, respectively, are both contained in $\mathscr{B}_{B}\left(c \log _{q}\left[K_{0}^{\prime}: K^{\prime}\right]+d\right)$. However, $\left(\pi(g) v, v^{\vee}\right)=0$ whenever the support of $\pi(g) v$ is disjoint from the support of $v^{\vee}$, or equivalently whenever $g \notin\left(S^{\vee}\right)^{-1} S$. Observing that there exists a positive constant $c_{1}$ such that $\mathscr{B}(N)^{-1} \mathscr{B}(N) \subset \mathscr{B}\left(c_{1} N\right)$ for all $N>0$, we conclude that the support of the matrix coefficient $\left(\pi(g) v, v^{\vee}\right)$ is contained in $\mathscr{B}\left(c_{1} c \log _{q}\left[K_{0}^{\prime}: K^{\prime}\right]+c_{1} d\right)$.

Remark 11. The proof shows also that in the global situation of a reductive group $\boldsymbol{G}$ defined over a number field $k$, there exist uniform constants $c$ and $d$ such that the assertion of the corollary is true for all local groups $\boldsymbol{G}\left(k_{v}\right), v$ a nonarchimedean place of $k$, and maximal compact subgroups $K_{0, v}$ that are hyperspecial for almost all $v$. One only needs to observe that every maximal compact subgroup of $G / T_{G}$ is conjugate to a maximal compact subgroup $\tilde{L}$ containing a fixed Iwahori subgroup $I$ [Tits 1979, §3.7]. Moreover, the index [ $\tilde{L}: I]$ is bounded by $q^{N}$, where $N$ does not depend on $v$. From this, we deduce that the constant $n_{0}$ in the proof of Lemma 8 can be bounded independently of $v$, if the norm on $U_{0}=\boldsymbol{U}_{0}\left(k_{v}\right)$ used in the proof is induced from the choice of a fixed $\mathbb{O}_{k}$-lattice in the Lie algebra of $\boldsymbol{U}_{0}$. The boundedness of all other constants is clear.

Remark 12. The maximal ocmes of $\mathrm{GL}(r, F)$ are (up to conjugation) parametrized by divisors of $r$. They can be realized as stabilizers of sequences $L_{i}, i \in \mathbb{Z}$, of O-lattices in $F^{r}$ such that $L_{i+l}=\varpi L_{i}$ and $\operatorname{dim}_{\mathbb{F}_{q}} L_{i} / L_{i+1}=k$ for all $i$, where $k$ is a divisor of $r$ and $k l=r$. Note that this stabilizer is the semidirect product of the parahoric subgroup of type $(k, \ldots, k)$ with the cyclic group generated by an element $z_{l}$ of GL $(r, F)$ with $z_{l} L_{i}=L_{i+1}$ [Carayol 1984]. 
Corollary 13. Assume that every supercuspidal representation of $G$ is contained in a representation induced from a cuspidal representation of an ocmc. Then $G$ has property (PSC). In particular, the following groups have property (PSC):

(1) $G=\mathrm{GL}(r, F)$ [Bushnell and Kutzko 1993a],

(2) $G=\mathrm{SL}(r, F)$ [Bushnell and Kutzko 1993b],

(3) $\boldsymbol{G}(F)$ for classical groups $\boldsymbol{G}$, provided $p \neq 2$ [Stevens 2008], and

(4) $\boldsymbol{G}\left(k_{v}\right)$ for any reductive group $\boldsymbol{G}$ defined over a number field $k$ and almost all nonarchimedean places $v$ of $k$ [Kim 2007]. Moreover, if the maximal compact subgroups $K_{0, v}$ of $\boldsymbol{G}\left(k_{v}\right)$ are hyperspecial for almost all $v$, then there are uniform constants $c$ and $d$ for which $\boldsymbol{G}\left(k_{v}\right)$ has property (PSC) with respect to $K_{0, v}$ for almost all $v$.

Remark 14. A general finiteness theorem of Bernstein [Bernstein 1974] (see also [Bernstein and Zelevinskii 1976; Bushnell 1990, p. 110]) shows (without appealing to any classification results) that for any open subgroup $K$ of $K_{0}$, there are, up to twisting by unramified characters, only finitely many supercuspidal representations $\pi$ of $G$ with a nontrivial $K$-fixed vector. Therefore, there necessarily exists a number $N=N(K)$ such that the support of all matrix coefficients $\left(\pi(g) v, v^{\vee}\right)$, $v \in \pi^{K}, v^{\vee} \in\left(\pi^{\vee}\right)^{K}$, is contained in $\mathscr{B}(N)$. To prove property (PSC) predicted by Conjecture 3 this way, it seems necessary to obtain an effective version of Bernstein's stabilization theorem (see [Bushnell 2001, Theorem 1]) with a realistic bound for the exponent $n_{K}$, namely a bound that is logarithmic in $\left[K_{0}^{\prime}: K^{\prime}\right]$.

\section{A class of parabolic subgroups}

Definition 15. We say that a maximal parabolic subgroup $\boldsymbol{P}=\boldsymbol{M U}$ is nice if there exists a positive constant $c$ such that for all $n>0$, we have

$$
\bar{U} \cap U Z(M) \mathscr{B}(n) \subset \begin{cases}\mathscr{B}(c n) \cup P w_{0} K_{n} & \text { if } w_{0} \boldsymbol{M} w_{0}^{-1}=\boldsymbol{M}, \\ \mathscr{B}(c n), & \text { otherwise. }\end{cases}
$$

In other words, $\boldsymbol{P}$ is nice if in a precise quantitative sense, for a compact subset $\Omega$ of $G$, either $\bar{U} \cap U Z(M) \Omega$ is bounded in terms of $\Omega$, or $\boldsymbol{P}^{w_{0}}=\overline{\boldsymbol{P}}$ and for a small open compact subgroup $K=K(\Omega)$ of $G$ the set $\bar{U} \cap U Z(M) \Omega \backslash P w_{0} K$ is bounded in terms of $\Omega$.

Our main result concerning this property is the following.

Proposition 16. Suppose that $\boldsymbol{G}$ is split and $\boldsymbol{U}$ is abelian. Then $\boldsymbol{P}$ is nice. Moreover, if $\boldsymbol{G}$ is defined and split over a number field $k$, then there is a uniform constant $c>0$ such that (2) is satisfied for all local groups $\boldsymbol{G}\left(k_{v}\right)$, where $v$ is a nonarchimedean place of $k$. 
The assumption that $\boldsymbol{G}$ is split is mainly for convenience and can probably be suppressed. For the convenience of the reader, we first present a proof in the case of $\boldsymbol{G}=\mathrm{GL}(r)$, where we can simplify the argument by direct matrix computations. The general case will be dealt with in Section 7 below.

Lemma 17. For $\boldsymbol{G}=\mathrm{GL}(r)$, all maximal parabolic subgroups are nice.

Proof. To fix ideas, we define the norm of elements of $G$ and the sets $\mathscr{B}(n)$ with respect to the standard 0 -lattice in $\mathfrak{g}$ spanned by the elementary matrices. With this normalization, we will obtain (2) for $c=2(r+1)$. For a matrix $X$ over $F$ we write $\|X\|$ (to be distinguished from $\|g\|_{G}$ for invertible $g$ ) for the standard norm of $X$, that is, the maximum of the absolute values of its entries.

Let $P$ be of type $\left(m^{\prime}, m\right)$. We may assume without loss of generality that $m \geq m^{\prime}$, for otherwise we can apply the automorphism $g \mapsto w_{0}{ }^{t} g^{-1} w_{0}$ of $\boldsymbol{G}$. Let

$$
\bar{u}=\left(\begin{array}{cc}
I_{m^{\prime}} & \\
X & I_{m}
\end{array}\right)
$$

and suppose that

$$
\bar{u}=\left(\begin{array}{cc}
\lambda I_{m^{\prime}} & * \\
& \mu^{-1} I_{m}
\end{array}\right) g, \quad \lambda, \mu \in F^{*}, \quad g=\left(\begin{array}{cc}
\alpha & \beta \\
\gamma & \delta
\end{array}\right) \in \mathscr{B}(n) .
$$

Note that $\|\bar{u}\|_{G} \leq\|X\|^{2}$. Modifying $g$ by a central element (and modifying $\lambda$ and $\mu$ accordingly), we can assume that $1 \leq|\operatorname{det} g|<q^{r}$. Then it is easy to see that the absolute values of the entries of $g$ are bounded by $q^{n}$. Note that $\gamma=\mu X$ and $\delta=\mu I_{m}$. In particular, we have $\|X\| \leq q^{n}|\mu|^{-1}$.

Suppose first that $m>m^{\prime}$. Expanding det $g$ as an alternating sum of products of entries of $g$, we see that each product contains at least one entry (in fact, at least $m-m^{\prime}$ entries) from $\delta$ as a factor. Thus $1 \leq|\operatorname{det} g| \leq q^{(r-1) n}|\mu|$, which implies $|\mu| \geq q^{-(r-1) n}$, and therefore $\|X\| \leq q^{r n}$ and $\|\bar{u}\|_{G} \leq q^{2 r n}$.

Suppose now that $m=m^{\prime}$. We distinguish the two cases $|\mu|>q^{-r n}$ and $|\mu| \leq q^{-r n}$. In the first case, we have $\|X\| \leq q^{(r+1) n}$ and $\|\bar{u}\|_{G} \leq q^{2(r+1) n}$. Assume therefore that $|\mu| \leq q^{-r n}$. The products in the expansion of det $g$ which do not contain an entry from $\delta$ as a factor add up to $(-1)^{m} \operatorname{det} \beta \operatorname{det} \gamma$. Therefore,

$$
\left|\operatorname{det} g-(-1)^{m} \operatorname{det} \beta \gamma\right| \leq|\mu| q^{(r-1) n} \leq q^{-n} .
$$

On the other hand, we have $|\operatorname{det} g| \geq 1$. Therefore $|\operatorname{det} g|=|\operatorname{det} \beta \gamma|$. In particular, $\gamma$ is invertible and

$$
|\operatorname{det} \gamma|^{-1}=|\operatorname{det} \beta \gamma|^{-1}|\operatorname{det} \beta| \leq|\operatorname{det} g|^{-1} q^{m n} \leq q^{m n} .
$$

It follows that $X$ is invertible and

$$
\left\|X^{-1}\right\|=|\mu|\left\|\gamma^{-1}\right\| \leq|\mu||\operatorname{det} \gamma|^{-1}\|\gamma\|^{m-1} \leq|\mu| q^{(r-1) n} \leq q^{-n} .
$$


Finally, the identity

$$
\bar{u}=\left(\begin{array}{cc}
X^{-1} & I_{m} \\
& X
\end{array}\right)\left(\begin{array}{cc} 
& -I_{m} \\
I_{m} &
\end{array}\right)\left(\begin{array}{cc}
I_{m} & X^{-1} \\
& I_{m}
\end{array}\right)
$$

shows that $\bar{u} \in P w_{0} K_{n}$.

Remark 18. While there are other cases of nice parabolic subgroups (for example, the maximal parabolic subgroups of $\mathrm{Sp}(4))$, unfortunately not all maximal parabolic subgroups are nice. As an example, consider

$$
\boldsymbol{G}=\operatorname{Sp}(6)=\left\{g \in \mathrm{GL}(6): g\left({ }_{-1}^{-1}{ }^{1}{ }^{1}\right) g^{t}=\left({ }_{-1}^{-1}{ }^{1}{ }^{1}\right)\right\}
$$

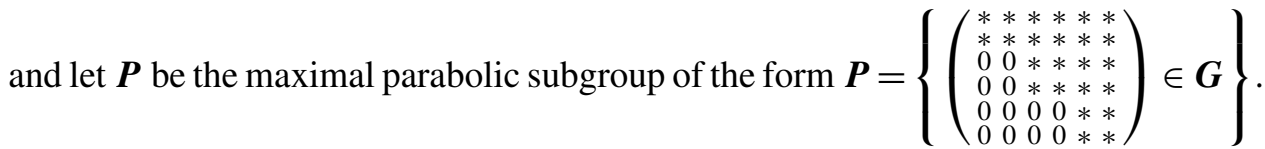

The equality

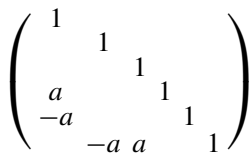

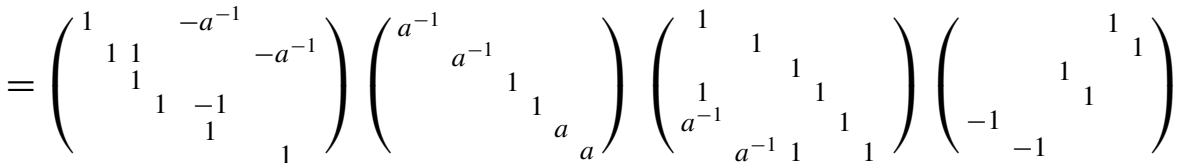

shows that

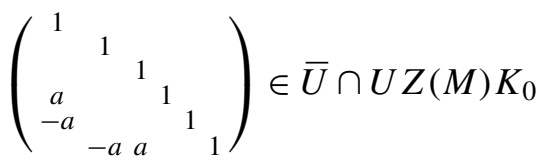

for all $a \in F$. However, if $\left(\begin{array}{ccc}* & * & * \\ * & * & * \\ A & B & C\end{array}\right) \in P w_{0} K_{n}$ (with blocks of size $2 \times 2$ ), then $\left\|A^{-1} B\right\| \leq q^{-n}$.

\section{Matrix coefficients of intertwining operators}

We now consider Conjectures 1 and 2 stated in the introduction, and prove some results in this direction. In particular, we prove Theorem 1.

Definition 19. Let $\boldsymbol{P}$ be a maximal parabolic subgroup of $\boldsymbol{G}$. We say $G$ has polynomial growth of matrix coefficients of intertwining operators (PIO) with respect to $P$ if there exist constants $c$ and $d$ such that for any open subgroup $K \subset K_{0}$ and any irreducible representation $\pi$ of $M$, the degrees of the numerators of the linear operators $M_{\bar{P} \mid P}(\pi, s)^{K}$ are bounded by $c \log _{q}\left[K_{0}^{\prime}: K^{\prime}\right]+d$. 
If this property is satisfied for all supercuspidal irreducible representations $\pi$ of $M$, we say that $G$ has polynomial growth of supercuspidal matrix coefficients of intertwining operators (PSIO) with respect to $P$.

Conjecture 1 amounts to the assertion that every $p$-adic reductive group $G$ satisfies property (PIO). It is easy to see that we can replace (PIO) by the weaker condition (PSIO). More precisely, we have the following.

Lemma 20. Suppose that any Levi subgroup $L \supset M_{0}$ of $G$ (including $G$ itself) satisfies (PSIO). Then G satisfies (PIO).

Proof. We argue as in the proof of Proposition 5. Let $\pi$ be an irreducible representation of $M$. By the Jacquet subrepresentation theorem, we can embed $\pi$ in an induced representation $I_{Q \cap M}^{M}(\sigma)$ for a parabolic subgroup $\boldsymbol{Q} \subset \boldsymbol{P}$ of $\boldsymbol{G}$ with Levi subgroup $\boldsymbol{L} \subset \boldsymbol{M}$ and an irreducible supercuspidal representation $\sigma$ of $L$. Consider the intertwining operators $M_{S_{2} \mid S_{1}}(\sigma, \lambda): I_{S_{1}}(\sigma, \lambda) \rightarrow I_{S_{2}}(\sigma, \lambda)$, $\lambda \in \mathfrak{a}_{L, \mathbb{C}}^{*}$, for parabolic subgroups $S_{1}, S_{2} \in \mathscr{P}(\boldsymbol{L})$. The embedding of $\pi$ into $I_{Q \cap M}^{M}(\sigma)$ gives rise to an embedding of $I_{P}(\pi, s)$ into $I_{Q}\left(\sigma, s \chi_{P}\right)$, and the restriction of $M_{\bar{Q} \mid Q}\left(\sigma, s \chi_{P}\right)$ to $I_{P}(\pi, s)$ becomes $M(\pi, s)$. We will bound the degrees of the matrix coefficients of $M\left(\sigma, s \chi_{P}\right)^{K}$. Let $\boldsymbol{Q}=\boldsymbol{Q}_{0}, \boldsymbol{Q}_{1}, \ldots, \boldsymbol{Q}_{l}=\overline{\boldsymbol{Q}}$ be a sequence of adjacent parabolic subgroups from $\boldsymbol{Q}$ to $\overline{\boldsymbol{Q}}$, and suppose that $\Delta_{\boldsymbol{Q}_{i}} \cap \Delta_{\boldsymbol{Q}_{i+1}}=\left\{\alpha_{i}\right\}$. We can decompose $M\left(\sigma, s \chi_{P}\right)$ into a product of rank-one intertwining operators $M_{Q_{i+1} \mid Q_{i}}\left(\sigma, s\left\langle\chi_{P}, \alpha_{i}^{\vee}\right\rangle\right)$. Therefore, it is enough to consider the degrees of the matrix coefficients of $M_{Q_{i+1} \mid Q_{i}}\left(\sigma, s\left\langle\chi_{P}, \alpha_{i}^{\vee}\right\rangle\right)^{K}, i=0, \ldots, l-1$. Fix $i$ and let $R=M_{R} N_{R}$ be the parabolic subgroup generated by $Q_{i}$ and $\boldsymbol{Q}_{i+1}$. Let $\boldsymbol{Q}^{\prime}=\boldsymbol{M}_{\boldsymbol{R}} \cap \boldsymbol{Q}_{i}$ and $\boldsymbol{Q}^{\prime \prime}=\boldsymbol{M}_{\boldsymbol{R}} \cap \boldsymbol{Q}_{i+1}$. Then $\boldsymbol{Q}^{\prime}$ and $\boldsymbol{Q}^{\prime \prime}$ are maximal parabolic subgroups of $\boldsymbol{M}_{\boldsymbol{R}}$ with Levi subgroup $\boldsymbol{L}$ and $\boldsymbol{Q}^{\prime \prime}=\overline{\boldsymbol{Q}^{\prime}}$. By [Waldspurger 2003, p. 284, (14)], the matrix coefficients of $M_{Q_{i+1} \mid Q_{i}}\left(\sigma, s\left\langle\chi_{P}, \alpha_{i}^{\vee}\right\rangle\right)^{K}$ are given by those of $M_{\overline{Q^{\prime}} \mid Q^{\prime}}\left(\sigma, s\left\langle\chi_{P}, \alpha_{i}^{\vee}\right\rangle\right)^{K \cap M_{R}}$. The lemma follows.

Theorem 21. Suppose that $\boldsymbol{P}=\boldsymbol{M U}$ is a nice maximal parabolic subgroup of $\boldsymbol{G}$ and that $M$ satisfies property (PSC). Then $G$ satisfies (PSIO) with respect to $P$.

Proof. Let $\pi$ be a supercuspidal representation of $M$. Assume that $K^{\prime}=K_{n}^{\prime}, n>0$, a normal subgroup of $K_{0}$. Let

$$
\varphi \in I_{P \cap K_{0}}^{K_{0}}\left(\left.\pi\right|_{M \cap K_{0}}\right)^{K_{n}^{\prime}} \quad \text { and } \quad \varphi^{\vee} \in I_{\bar{P} \cap K_{0}}^{K_{0}}\left(\left.\pi^{\vee}\right|_{M \cap K_{0}}\right)^{K_{n}^{\prime}} .
$$

This is equivalent to $\varphi(k) \in \pi^{M \cap K_{n}^{\prime}}$ and $\varphi^{\vee}(k) \in\left(\pi^{\vee}\right)^{M \cap K_{n}^{\prime}}$ for all $k \in K_{0}$. We extend these functions to functions $\varphi_{s} \in I_{P}(\pi, s)$ and $\varphi_{s}^{\vee} \in I_{\bar{P}}\left(\pi^{\vee}, s\right)$. Then the matrix coefficient $\left(M(\pi, s) \varphi_{s}, \varphi_{s}^{\vee}\right)$ can be computed as

$$
\left(M(\pi, s) \varphi_{s}, \varphi_{s}^{\vee}\right)=\int_{K_{0}}\left(\left(M(\pi, s) \varphi_{s}\right)(k), \varphi^{\vee}(k)\right) d k=\int_{\bar{U}}\left|\chi_{P}\right|(\bar{u})^{s} f(\bar{u}) d \bar{u},
$$


with

$$
f(\bar{u})=\int_{K_{0}}\left(\varphi_{0}(\bar{u} k), \varphi^{\vee}(k)\right) d k .
$$

Note that $f$ is right $\bar{U} \cap K_{n}^{\prime}$-invariant. Since $M$ satisfies property (PSC), there is a constant $c_{1}>0$ such that the matrix coefficients $\left(\pi(m) \varphi\left(k^{\prime}\right), \varphi^{\vee}(k)\right), m \in M$, $k, k^{\prime} \in K_{0}$, all vanish for $m \notin \mathscr{B}^{M}\left(c_{1} n\right)$. Furthermore, there exists a constant $c_{2}>0$ with $\mathscr{B}^{M}(l) \subset Z(M) \mathscr{B}_{(}\left(c_{2} l\right)$ for all $l>0$. Applying the Iwasawa decomposition to $\bar{u}$, it follows that the support of $f$ is contained in $\bar{U} \cap U Z(M) \mathscr{B}_{(}\left(c_{1} c_{2} n\right)$. Consider first the case where $\boldsymbol{P}^{w_{0}} \neq \overline{\boldsymbol{P}}$. Because $\boldsymbol{P}$ is nice, we conclude from the above that the support of $f$ is contained in $\bar{U} \cap \mathscr{S}\left(c c_{1} c_{2} n\right)$ for the constant $c$ of Definition 15 . Thus, up to a constant, the integral becomes a finite sum

$$
\sum_{\bar{u} \in \bar{U} \cap \Re\left(c c_{1} c_{2} n\right) / \bar{U} \cap K_{n}^{\prime}}\left|\chi_{P}\right|(\bar{u})^{s} f(\bar{u})
$$

which is a polynomial in $q^{-s}$ of degree at most $-\log _{q} \min _{\bar{U} \cap \Re\left(c c_{1} c_{2} n\right)}\left|\chi_{P}\right| \ll n$.

We still need to consider the case $\boldsymbol{P}^{w_{0}}=\overline{\boldsymbol{P}}$. Let $\omega_{\pi}$ be the central character of $\pi$. We take an element $a \in Z(M)$ as follows. If

$$
\left.\left.\omega_{\pi}\right|_{Z(M)^{1}} \not \equiv \omega_{w_{0} \pi}\right|_{Z(M)^{1}}
$$

then we take any $a \in Z(M)^{1}=Z(M) \cap K_{0}$ such that $\omega_{\pi}(a) \neq \omega_{\pi}(b)$ where $b=w_{0}^{-1} a w_{0} \in Z(M)$. Otherwise we take $a$ which generates $T_{0} \cap Z(M)$ modulo $Z(G) Z(M)^{1}$ and for which $\left|\chi_{P}\right|(a)=|\alpha(a)|^{\frac{1}{2}}=q^{-m}<1$. We have $m \in \frac{1}{2} \mathbb{Z}_{>0}$.

We take $n_{0} \geq 0$ such that $K_{n}^{\prime} \cap b K_{n}^{\prime} b^{-1} \supset K_{n+n_{0}}^{\prime}$ and $Z(G) K_{n}^{\prime} \supset Z(G) K_{n+n_{0}}$ for all $n$.

Note that under the action of $K_{0}$ the space $I_{\bar{P} \cap K_{0}}^{K_{0}}\left(\left.\pi^{\vee}\right|_{M \cap K_{0}}\right)^{K_{n+n_{0}}^{\prime}}$ is spanned by functions $\varphi^{\vee}$ with support $\left(P \cap K_{0}\right) K_{n+n_{0}}^{\prime}$. Thus, we can assume that $\varphi^{\vee}$ has this property. Hence, $\varphi^{\vee}$ is determined by its value at the identity and

$$
\left(M(\pi, s) \varphi_{s}, \varphi_{s}^{\vee}\right)=c\left(M(\pi, s) \varphi_{s}(e), \varphi^{\vee}(e)\right)=c \int_{\bar{U}}\left|\chi_{P}\right|(\bar{u})^{s}\left(\varphi_{0}(\bar{u}), \varphi^{\vee}(e)\right) d \bar{u}
$$

for some constant $c$. If $\varphi$ vanishes at $w_{0}$, then the last integrand vanishes on $\bar{U} \cap P w_{0} K_{n}^{\prime} \supset \bar{U} \cap P w_{0} K_{n+n_{0}}$, and we can argue as in the case $\boldsymbol{P}^{w_{0}} \neq \overline{\boldsymbol{P}}$ above.

Otherwise, observe that

$$
\begin{aligned}
\left(M(\pi, s) I_{P}(b, s) \varphi_{s}, \varphi_{s}^{\vee}\right) & =\left(I_{\bar{P}}(b,-s) M(\pi, s) \varphi_{s}, \varphi_{s}^{\vee}\right) \\
& =c\left(M(\pi, s) \varphi_{s}(b), \varphi^{\vee}(e)\right)=\delta_{\bar{P}}^{\frac{1}{2}}(b) \omega_{s}(b)\left(M(\pi, s) \varphi_{s}, \varphi_{s}^{\vee}\right) \\
& =\delta_{P}^{\frac{1}{2}}(a) \omega_{s}(b)\left(M(\pi, s) \varphi_{s}, \varphi_{s}^{\vee}\right),
\end{aligned}
$$


where $\omega_{s}$ is the character $\omega_{\pi}\left|\chi_{\bar{P}}\right|^{-s}=\omega_{\pi}\left|\chi_{P}\right|^{s}$ of $Z(M)$. Thus, if we consider the operator

$$
\Delta_{a, s}=\omega_{s}\left(b^{-1}\right) \delta_{P}^{-\frac{1}{2}}(a) I(b, s)-\omega_{s}\left(b^{-1} a\right) \mathrm{Id}
$$

on $I_{P}(\pi, s)$ then $\Delta_{a, s} \varphi_{s}$ vanishes at $w_{0}$, while

$$
\left(M(\pi, s) \Delta_{a, s} \varphi_{s}, \varphi_{s}^{\vee}\right)=\left(1-\omega_{s}\left(b^{-1} a\right)\right)\left(M(\pi, s) \varphi_{s}, \varphi_{s}^{\vee}\right) .
$$

If condition (3) holds then

$$
\left(M(\pi, s) \varphi_{s}, \varphi_{-s}^{\vee}\right)=\left(1-\omega_{\pi}\left(b^{-1} a\right)\right)^{-1}\left(M(\pi, s) \Delta_{a, s} \varphi_{s}, \varphi_{s}^{\vee}\right),
$$

and since $\Delta_{a, s} \varphi_{s} \in I_{P}(\pi, s)^{K_{n}^{\prime}}$, we reduce to the previous case. Otherwise,

$$
\left(M(\pi, s) \varphi_{s}, \varphi_{-s}^{\vee}\right)=\left(1-\omega_{\pi}\left(b^{-1} a\right) q^{-2 m s}\right)^{-1}\left(M(\pi, s) \Delta_{a, s} \varphi_{s}, \varphi_{s}^{\vee}\right)
$$

and $\Delta_{a, s} \varphi_{s} \in I_{P}(\pi, s)^{K_{n+n_{0}}^{\prime}}$. So once again, we reduce to the previous case.

Remark 22. The argument also gives a simple proof of the rationality of $M(\pi, s)$ for supercuspidal $\pi$ and nice $\boldsymbol{P}$. More precisely, it shows that $M(\pi, s)$ is a polynomial in $q^{-s}$ if either $\boldsymbol{P}^{w_{0}} \neq \overline{\boldsymbol{P}}$ or $\left.\omega_{\pi} \omega_{w_{0} \pi}^{-1}\right|_{Z(M)^{1}} \neq 1$. Otherwise,

$$
\left(1-\omega_{\pi}\left(w_{0}^{-1} a^{-1} w_{0} a\right) q^{-2 m s}\right)
$$

is a polynomial in $q^{-s}$, where $a$ and $m$ are as above.

Remark 23. In the global situation of Conjecture 2, the proof shows that the constants $c$ and $d$ appearing in the definition of property (PSIO) can be chosen independently of the nonarchimedean place $v$, if this is the case for the constants appearing in Definition 7 (definition of property (PSC)) and Definition 15. By the fourth part of Corollary 13, for property (PSC) this uniformity statement is always satisfied after omitting finitely many places. Uniformity of the constant in Definition 15 is satisfied in the cases covered by Proposition 16.

Proof of Theorem 1. Lemma 17 and Corollary 13 show that in the case of $\boldsymbol{G}=\mathrm{GL}(r)$, the conditions of Theorem 21 hold for all maximal parabolic subgroups of $\boldsymbol{G}$. Therefore, $G$ satisfies property (PSIO). Lemma 20 finishes the argument. The assertion on the constants $c$ and $d$ is clear.

\section{Parabolic subgroups with abelian unipotent radical}

In this section, we prove Proposition 16 in general. Parabolic subgroups with Abelian unipotent radical and the associated action of their Levi subgroup on the radical have been studied by Richardson, Röhrle and Steinberg [1992]. We recall their results and extend them as necessary. 
Let $\boldsymbol{G}$ be a split reductive group over $F$. It will be convenient to write $\mathfrak{g}$ in terms of a Chevalley basis [Serre 2001]. Namely, choose $X_{\alpha} \in \mathfrak{g}_{\alpha}, \alpha \in \Phi=R\left(\boldsymbol{T}_{0}, \boldsymbol{G}\right)$, such that

$$
\left[X_{\alpha}, X_{\beta}\right]= \begin{cases}N_{\alpha, \beta} X_{\alpha+\beta} & \text { if } \alpha+\beta \in \Phi, \\ H_{\alpha} & \text { if } \alpha=-\beta \\ 0 & \text { otherwise. }\end{cases}
$$

Here, the structure constants $N_{\alpha, \beta}, \alpha, \beta, \alpha+\beta \in \Phi$, satisfy $N_{\alpha, \beta}= \pm(p+1)$, where $p$ is the largest integer with $\beta-p \alpha \in \Phi$.

Obviously, to prove Proposition 16 we can pass to the adjoint group, which is a direct product of simple groups. Therefore, suppose from now on that $\boldsymbol{G}$ is simple and adjoint, $\boldsymbol{P}$ is maximal, and $\boldsymbol{U}$ is abelian. (Actually, the maximality of $\boldsymbol{P}$ is then automatic.) Let $K_{0}$ be the stabilizer of the 0 -lattice spanned by the Chevalley basis, which is a hyperspecial maximal compact subgroup of $G$. Let $\alpha$ be the simple root defining $\boldsymbol{P}$. Write $\mathfrak{m}=\operatorname{Lie} M, \mathfrak{u}=\operatorname{Lie} U$, and $\overline{\mathfrak{u}}=\operatorname{Lie} \bar{U}$, so that $\mathfrak{g}=\mathfrak{u} \oplus \mathfrak{m} \oplus \overline{\mathfrak{u}}$. Denote by $\Phi_{U}=R\left(\boldsymbol{T}_{0}, \boldsymbol{U}\right)$ the roots in $\mathfrak{u}$, namely the roots whose $\alpha$-coefficient in the expansion with respect to $\Delta_{0}$ is positive. (Since $\boldsymbol{U}$ is abelian, this coefficient is necessarily 1.) Let $\rho$ be the highest root. We have $\alpha, \rho \in \Phi_{U}$. The roots orthogonal to $\rho$ form a parabolic root subsystem $\Phi_{1}$ which contains a unique irreducible constituent $\Phi_{1}^{\prime} \supset \Phi_{U} \cap \Phi_{1}$. If $\boldsymbol{G}$ is not simply laced, we write $\rho_{s}$ for the highest short root and $\delta=\rho-\rho_{s}=-s_{\rho} \rho_{s} \in \Phi$. We have $\rho_{s}, 2 \rho_{s}-\rho=-s_{\rho_{s}} \rho \in \Phi_{U}$.

Lemma 24. Suppose that $\boldsymbol{G}$ is not simply laced and let $\rho, \rho_{s}$ and $\delta$ be as before. Then the following conditions are equivalent for $\gamma \in \Phi_{U}$ :

(1) $\gamma+\delta, \gamma+2 \delta \in \Phi_{U}$.

(2) $\gamma$ is long and $\left\langle\delta, \gamma^{\vee}\right\rangle=-1$.

(3) $\gamma$ is long, $\left\langle\rho, \gamma^{\vee}\right\rangle=0$, and $\left\langle\rho_{s}, \gamma^{\vee}\right\rangle=1$.

(4) $\gamma$ is the highest root in $\Phi_{1}^{\prime}$.

(5) $\gamma=2 \rho_{s}-\rho$.

Proof. The first three conditions are clearly equivalent and they hold for $\gamma=2 \rho_{s}-\rho$. It remains to consider the cases of $B_{n}$ and $C_{n}$. In the $B_{n}$ case $\rho=2 \epsilon_{1}, \rho_{s}=\epsilon_{1}+\epsilon_{2}$, $\delta=\epsilon_{1}-\epsilon_{2}, \gamma=2 \epsilon_{2}$. In the $C_{n}$ case $\rho=\epsilon_{1}+\epsilon_{2}, \rho_{s}=\epsilon_{1}, \delta=\epsilon_{2}, \gamma=\epsilon_{1}-\epsilon_{2}$.

We fix once and for all a tuple $\left(\beta_{1}, \ldots, \beta_{r}\right)$ of mutually orthogonal long roots in $\Phi_{U}$ with $r$ maximal.

Theorem 25 [Richardson et al. 1992, Theorem 2.1]. (1) For any $0 \leq s \leq r$, the Weyl group of $\boldsymbol{M}$ acts transitively on the set of s-tuples of mutually orthogonal long roots in $\Phi_{U}$. 
(2) Fix $u_{i} \in U_{\beta_{i}} \backslash\{0\}$. Then $\left\{\prod_{i=1}^{s} u_{i}\right\}_{s=0}^{r}$ is a set of representatives for the $\boldsymbol{M}$ orbits in $\boldsymbol{U}$ under the conjugation action. (The integer $s$ is called the rank of the orbit.)

The orbit corresponding to $s=r$ is the open orbit of the $\boldsymbol{M}$-action on $\boldsymbol{U}$. It is the intersection with $\boldsymbol{U}$ of the Richardson orbit associated to $\boldsymbol{P}$. The orbit corresponding to $s=0$ is the zero orbit.

Remark 26. The possibilities (up to isogeny) for $\boldsymbol{G}$ and $\boldsymbol{P}$ have been enumerated in [Richardson et al. 1992, Remark 2.3], and the corresponding values of $r$ are listed in [Richardson et al. 1992, Table 1]. We can explicate the orbit classification of Theorem 25 case by case.

In the cases where $\boldsymbol{G}=\mathrm{GL}(m), \boldsymbol{M}=\mathrm{GL}(k) \times \mathrm{GL}(m-k), \boldsymbol{U}$ is the space of $k \times(m-k)$ matrices, and $0<k<m$, or $\boldsymbol{G}=\mathrm{Sp}(2 m), \boldsymbol{M}=\mathrm{GL}(m)$, and $\boldsymbol{U}$ is the space of symmetric $m \times m$ matrices, the notion of rank given by Theorem 25 coincides with the usual notion for matrices. In the case $\boldsymbol{G}=\mathrm{SO}(2 m), \boldsymbol{M}=\mathrm{GL}(m)$, and $\boldsymbol{U}$ is the space of antisymmetric $m \times m$ matrices, the rank in our sense is one half of the rank of the matrix. In the case $\boldsymbol{G}=\mathrm{SO}(m), \boldsymbol{M}=\mathrm{GL}(1) \times \mathrm{SO}(m-2)$, and $\boldsymbol{U}$ is a quadratic space of dimension $m-2$, the rank is one for a nonzero isotropic vector and two for anisotropic vectors.

There are (up to automorphisms of $\boldsymbol{G}$ ) two exceptional cases. For $\boldsymbol{G}=E_{6}$, $\boldsymbol{M}=\mathrm{GSpin}(10)$, and $\boldsymbol{U}$ one of the 16-dimensional half-spin representations of $\boldsymbol{M}$, we have $r=2$. The nonzero pure spinors (i.e., the spinors in the orbit of 1 , the unit element of the exterior algebra) have rank one, and the remaining nonzero spinors have rank two. The orbit dimensions are 0,11, and 16, respectively [Igusa 1970, Proposition 2]. For $\boldsymbol{G}=E_{7}, \boldsymbol{M}=G E_{6}$, and $\boldsymbol{U}$ the 27-dimensional representation of $\boldsymbol{M}$, we have $r=3$. The derived group of $\boldsymbol{M}$ leaves a nonzero cubic form $f$ on $\boldsymbol{U}$ invariant, and this form is unique up to a scalar. The rank is one for the nonzero vectors in the singular locus of the hypersurface $f=0$, two for the remaining nonzero vectors with $f=0$, and three for the vectors with $f \neq 0$ [Chevalley 1951]. The orbit dimensions are $0,17,26$, and 27, respectively [Richardson et al. 1992, Table 2].

Note that the second part of Theorem 25 does not apply to the $M$-orbits in $U$. However, the proof of [Richardson et al. 1992, Theorem 2.1] (see also [loc. cit., Theorem 5.3]) shows that fixing $\beta_{1}, \ldots, \beta_{r}$ as above, it is still true that any $M$-orbit in $U$ of rank $s$ contains a representative of the form $\prod_{i=1}^{s} u_{i}$ for some $u_{i} \in U_{\beta_{i}} \backslash\{0\}$. More precisely, we have:

Lemma 27. Let $\beta_{1}, \ldots, \beta_{r}$ be as above. Then there exists a compact set $\omega \subset M$ with the following property: for all $X \in \mathfrak{u}$, there is $m \in \omega$ such that $\operatorname{Ad}(m) X$ is a linear combination of $X_{\beta_{1}}, \ldots, X_{\beta_{r}}$. If either $\boldsymbol{G}$ is simply laced or $p \neq 2$, then we can take $\omega=K_{M}=M \cap K_{0}$. 
Proof. Write $X=\sum_{\beta \in \Phi_{U}} c_{\beta}(X) X_{\beta}$. Let $\rho \in \Phi_{U}$ be the highest root. We follow the argument of [Richardson et al. 1992, Proposition 2.13]. The proof is by induction on the rank of $\boldsymbol{G}$. The case $X=0$ is trivial, so we assume that $X \neq 0$. The first step is to show that in the $\operatorname{Ad} K_{M}$-orbit of $X$, we can choose $X^{\prime}$ such that $\left|c_{\beta}\left(X^{\prime}\right)\right| \leq D\left|c_{\rho}\left(X^{\prime}\right)\right|$ for all $\beta \in \Phi_{U}$, where $D$ is a fixed constant which can be taken to be 1 if $p \neq 2$ or if $\boldsymbol{G}$ is simply laced. This is done as follows. Let $\beta_{0} \in \Phi_{U}$ be such that $\left|c_{\beta_{0}}(X)\right|$ is maximal. Applying a Weyl element of $M$, we can assume that either $\beta_{0}=\rho$ or $\beta_{0}=\rho_{s}$ (in the nonsimply laced case). If $\left|c_{\rho}(X)\right|=\left|c_{\beta_{0}}(X)\right|$ (and in particular, if $\boldsymbol{G}$ is simply laced), then we are done. Assume that this is not the case and let $\delta=\rho-\rho_{s}$ and $X^{\prime}=\operatorname{Ad}\left(u_{\delta}(t)\right) X$ with $t \in \mathcal{O}$. It follows from Lemma 24 and the commutation relations that

$$
c_{\gamma}\left(X^{\prime}\right)= \begin{cases}c_{\rho}(X) \pm 2 t c_{\rho_{s}}(X)+t^{2} c_{2 \rho_{s}-\rho}(X) & \text { if } \gamma=\rho, \\ c_{\gamma}(X) \pm t c_{\gamma-\delta}(X) & \text { if } \gamma \neq \rho \text { and } \gamma-\delta \in \Phi, \\ c_{\gamma}(X) & \text { if } \gamma-\delta \notin \Phi .\end{cases}
$$

Therefore, we can choose $t \in 0^{*}$ such that $\left|c_{\rho}\left(X^{\prime}\right)\right|=\max _{\beta \in \Phi_{U}}\left|c_{\beta}\left(X^{\prime}\right)\right|$ if $p \neq 2$ and $\left|c_{\rho}\left(X^{\prime}\right)\right| \geq \frac{1}{2}|2| \max _{\beta \in \Phi_{U}}\left|c_{\beta}\left(X^{\prime}\right)\right|$ if $p=2$.

The second step is to clear the coefficients of all roots which are not orthogonal to $\rho$ by conjugating by suitable unipotent elements. This is done as in [Richardson et al. 1992, p. 655], except that our condition on $X^{\prime}$ guarantees that the conjugating elements are taken from $K_{M}$ (or at least from a bounded set, if $p=2$ and $\boldsymbol{G}$ is not simply laced). The rest of the proof (the induction step) follows [loc. cit.].

Let $w=s_{\beta_{1}} \ldots s_{\beta_{r}}$. Note that the reflections $s_{\beta_{i}}$ commute with each other, since the roots $\beta_{i}$ are mutually orthogonal. For any $\beta \in \Phi_{U}$, let $\mathcal{N}(\beta)$ be the multiset

$$
\mathcal{N}(\beta)= \begin{cases}\left\{\beta_{i}:\left\langle\beta, \beta_{i}^{\vee}\right\rangle=1\right\} & \text { if } \beta \neq \beta_{1}, \ldots, \beta_{r}, \\ \left\{\beta_{i}, \beta_{i}\right\} & \text { if } \beta=\beta_{i} .\end{cases}
$$

Thus, $\mathcal{N}(\beta)$ consists of the roots $\beta_{i}$ which are not orthogonal to $\beta$, counted with multiplicity $\left\langle\beta, \beta_{i}^{\vee}\right\rangle$. Note that $w \beta=\beta-\sum \mathcal{N}(\beta)$ for any $\beta \in \Phi_{U}$. Also, for any $\beta \in \Phi_{U}$,

$$
|\mathcal{N}(\beta)|=\sum_{i=1}^{r}\left\langle\beta, \beta_{i}^{\vee}\right\rangle,
$$

and by [Richardson et al. 1992, Lemma 2.10], we have $1 \leq|\mathcal{N}(\beta)| \leq 2$.

Suppose that $\beta, \gamma \in \Phi_{U}$ are distinct and $\beta$ is long. Then the following conditions are equivalent:

(1) $\left\langle\gamma, \beta^{\vee}\right\rangle \neq 0$,

(2) $\left\langle\gamma, \beta^{\vee}\right\rangle=1$, 
(3) $\gamma-\beta \in \Phi$, and

(4) $\gamma-\beta=s_{\beta}(\gamma)$.

For any $X \in \mathfrak{u}$, denote by $D_{X}$ the double commutator map

$$
D_{X}=\left.\left.\frac{1}{2} \operatorname{ad} X\right|_{\mathfrak{m}} \circ \operatorname{ad} X\right|_{\overline{\mathfrak{u}}} \in \operatorname{Hom}_{F}(\overline{\mathfrak{u}}, \mathfrak{u}) .
$$

Analogously, for $\bar{X} \in \overline{\mathfrak{u}}$, we denote by $\bar{D}_{\bar{X}}$ the double commutator map

$$
\bar{D}_{\bar{X}}=\left.\left.\frac{1}{2} \operatorname{ad} \bar{X}\right|_{\mathfrak{m}} \circ \operatorname{ad} \bar{X}\right|_{\mathfrak{u}} \in \operatorname{Hom}_{F}(\mathfrak{u}, \overline{\mathfrak{u}}) .
$$

Lemma 28. Let $X=\sum_{i=1}^{r} t_{i} X_{\beta_{i}}$. Then

$$
D_{X} X_{-\beta}= \begin{cases}0 & \text { if }|\mathcal{N}(\beta)|=1 \\ t_{i} t_{j} X_{-w \beta} & \text { if } \mathcal{N}(\beta)=\left\{\beta_{i}, \beta_{j}\right\}\end{cases}
$$

Proof. The statement is clear if $\beta=\beta_{i}$, since $\beta_{i}-\beta_{j} \notin \Phi$ for all $j$.

Now suppose that $\beta \neq \beta_{1}, \ldots, \beta_{r}$. Then

$$
\operatorname{ad} X\left(X_{-\beta}\right)=\sum_{i: \beta_{i} \in \mathcal{N}(\beta)} t_{i} X_{\beta_{i}-\beta},
$$

and therefore

$$
D_{X}\left(X_{-\beta}\right)=\frac{1}{2} \sum_{\substack{i, j: \beta_{i} \in \mathcal{N}(\beta), \beta_{i}+\beta_{j}-\beta \in \Phi_{U}}} t_{i} t_{j} X_{\beta_{i}+\beta_{j}-\beta} .
$$

Note that if $\beta_{i} \in \mathcal{N}(\beta)$ and $\delta=\beta_{i}+\beta_{j}-\beta \in \Phi_{U}$, then $i \neq j$, since $\beta_{i}$ is long. If we set $\gamma=\beta_{i}-\beta=-s_{\beta_{i}} \beta$, then $\delta=\beta_{j}+\gamma$ and $s_{\beta_{i}} \delta=\beta_{j}-\beta \in \Phi$. Thus, $\beta_{j} \in \mathcal{N}(\beta)$ and $\delta=-w \beta$.

Corollary 29. For any $X \in \mathfrak{u}$, we have $\left\|D_{X}\right\|_{\operatorname{Hom}(\overline{\mathfrak{u}}, \mathfrak{u})} \gg\|X\|^{2}$.

Lemma 30. The following conditions are equivalent:

(1) $\boldsymbol{P}$ is conjugate to $\overline{\boldsymbol{P}}$.

(2) $\boldsymbol{P}^{w_{0}}=\overline{\boldsymbol{P}}$.

(3) $\boldsymbol{P}^{w}=\overline{\boldsymbol{P}}$.

(4) $|\mathcal{N}(\beta)|=2$ for all $\beta \in \Phi_{U}$.

(5) $\frac{1}{2} \sum_{i=1}^{r} \beta_{i}^{\vee}$ is the fundamental coweight with respect to $\boldsymbol{P}$.

(6) $\frac{1}{2} \sum_{i=1}^{r} \beta_{i}$ is the fundamental weight with respect to $\boldsymbol{P}$.

(7) There exists $X \in \mathfrak{u}$ such that $D_{X}$ is invertible.

If these conditions are satisfied, then $D_{X}$ is invertible if and only if $X$ belongs to the open Ad $\boldsymbol{M}$-orbit in $\mathfrak{u}$. 
Proof. The equivalence of the first four conditions follows from [Richardson et al. 1992, Proposition 3.12]. The equivalence of the last and the fourth conditions, as well as the last assertion of the lemma, follows from Lemma 28. The equivalence between the fourth and fifth conditions follows from (4). Finally, the equivalence between the fifth and the sixth conditions is immediate, since $\alpha$ is a long root.

Let $H$ be the central element of $\mathfrak{m}$ such that ad $\left.H\right|_{\mathfrak{u}}=2 \operatorname{Id}_{\mathfrak{u}}$.

Lemma 31. Suppose that $\boldsymbol{P}^{w_{0}}=\overline{\boldsymbol{P}}$. Then

(1) We have $H=\sum_{i=1}^{r} H_{\beta_{i}}$.

(2) The open $(P, P)$ Bruhat cell is $P w_{0} U$.

(3) We have

$$
P w_{0} U=\left\{g \in G:\left.\operatorname{proj}_{\overline{\mathfrak{u}}} \circ \operatorname{Ad}(g)\right|_{\mathfrak{u}} \text { is invertible }\right\} .
$$

(4) For any $g \in P w_{0} U$, the $U$-part in the Bruhat decomposition is given by $\exp Y$, where $2 Y=\left(\left.\operatorname{proj}_{\overline{\mathfrak{u}}} \circ \operatorname{Ad}(g)\right|_{\mathfrak{u}}\right)^{-1}\left(\operatorname{proj}_{\overline{\mathfrak{u}}}(\operatorname{Ad}(g) H)\right)$.

(5) In particular, for $\bar{X} \in \overline{\mathfrak{u}}$, we have $\exp \bar{X} \in P w_{0} U$ if and only if $\bar{X}$ lies in the open Ad $\boldsymbol{M}$-orbit, and in this case the $U$-part of $\exp \bar{X}$ is $\exp Y$ for $Y=\bar{D}_{\bar{X}}^{-1}(\bar{X})$.

Proof. The first part follows from the previous lemma. The second part is clear. Let $\mathscr{C}=\left\{g \in G:\left.\operatorname{proj}_{\overline{\mathfrak{u}}} \circ \operatorname{Ad}(g)\right|_{\mathfrak{u}}\right.$ is invertible $\}$. Clearly, $\mathscr{C}$ is left and right $P$-invariant and $w_{0} \in \mathscr{C}$. Therefore $\mathscr{C}$ is a union of $(P, P)$ double cosets and $P w_{0} U \subset \mathscr{C}$. The fourth part is also clear by direct computation. By [Richardson et al. 1992, Theorem 1.1], every $(P, P)$ double coset intersects $\bar{U}$ in (the set of $F$-rational points of) a single $\boldsymbol{M}$-orbit under conjugation. Thus, in order to show that $\mathscr{C}=P w_{0} U$, it is enough to show that $\mathscr{C} \cap \bar{U}$ is (the set of $F$-rational points of) an $\boldsymbol{M}$-orbit. However, $\mathscr{C} \cap \bar{U}=\left\{\exp \bar{X}: \bar{D}_{\bar{X}}\right.$ is invertible $\}$. Therefore, the statement follows from Lemma 30.

Corollary 32. Let $\theta$ be the Cartan involution of $\boldsymbol{G}$ and set $d=\#\left\{\beta \in \Phi_{U}: \beta_{i} \in\right.$ $\mathcal{N}(\beta)\}$, which is independent of $i$. If $\boldsymbol{P}^{w_{0}}=\overline{\boldsymbol{P}}$, then $d=2 \operatorname{dim} U / r$. For $X=$ $\sum_{i=1}^{r} t_{i} X_{\beta_{i}}$, we have

$$
\operatorname{det}\left(\theta \circ D_{X}\right)= \begin{cases}\left(t_{1} \ldots t_{r}\right)^{d} & \text { if } \boldsymbol{P}^{w}=\overline{\boldsymbol{P}}, \\ 0 & \text { otherwise. }\end{cases}
$$

Remark 33. Suppose that $\boldsymbol{P}^{w_{0}}=\overline{\boldsymbol{P}}$. The character $\prod_{i=1}^{r} \beta_{i}$ of $\boldsymbol{T}_{0}$ is trivial on $\boldsymbol{M}^{\prime}$ and therefore extends to a rational character $\psi$ of $\boldsymbol{M}$. The polynomial

$$
\sum_{i=1}^{r} t_{i} X_{\beta_{i}} \mapsto t_{1} \ldots t_{r}
$$

extends to an irreducible $(\operatorname{Ad} M, \psi)$-equivariant polynomial $\Delta$ on $\mathfrak{u}$. 
For $n \in N_{M}(T)$ representing $w \in W^{M}$ and $\beta \in \Phi_{U}$, let $f_{n, \beta}$ be the scalar so that $\operatorname{Ad}(n) X_{\beta}=f_{n, \beta} X_{w \beta}$. Clearly $f_{n t, \beta}=\beta(t) f_{n, \beta}$. In the simply laced case, we have

$$
\Delta\left(\sum_{\beta \in \Phi_{U}} c_{\beta} X_{\beta}\right)=\sum_{w \in N_{M}\left(T_{0}\right) / T_{0}} \psi\left(n_{w}\right) \frac{c_{w \beta_{1}}}{f_{n_{w}, \beta_{1}}} \ldots \frac{c_{w \beta_{r}}}{f_{n_{w}, \beta_{r}}}
$$

where $n_{w}$ is any representative of $w$ in $M$. The polynomial $\Delta$ is the determinant in the $\mathrm{GL}(m)$ or $\mathrm{Sp}(2 m)$ case, the Pfaffian in the $\mathrm{SO}(4 m)$ case, the canonical quadratic form in the $\mathrm{SO}(m)$ case, and the relatively invariant cubic form in the $E_{7}$ case.

Corollary 34. Assume that $\boldsymbol{P}^{w_{0}}=\overline{\boldsymbol{P}}$.

(1) The open orbit in $\mathfrak{u}$ is the principal open set defined by $\operatorname{det} \theta \circ D_{X}$.

(2) Assume that $X \in \mathfrak{u}$ is in the open orbit. Then the Jacobson-Morozov parabolic subgroup of $X$ is $\boldsymbol{P}$.

(3) Assume that $X=\sum_{i=1}^{r} t_{i} X_{\beta_{i}}$ with $t_{1}, \ldots, t_{r} \neq 0$. Let $\bar{X}=\sum_{i=1}^{r} t_{i}^{-1} X_{-\beta_{i}}$. Then $(X, H, \bar{X})$ is an SL(2)-triple.

Remark 35. In [Kac 1980], the double commutator map has been used to obtain relatively invariant polynomials in a more general situation.

Finally, we are ready to prove Proposition 16.

Proof of Proposition 16. Suppose that $\bar{u} \in \bar{U} \cap Z(M) U \Re(n)$ and write $\bar{u}=z u b$, where $z \in Z(M), u \in U$, and $b \in \mathscr{B}(n)$. Let $\lambda \in F^{*}$ be such that $\left.\operatorname{Ad}(z)\right|_{\mathfrak{u}}=\lambda \operatorname{Id}_{\mathfrak{u}}$. Also write $\bar{u}=\exp \bar{X}$, where $\bar{X} \in \bar{u}$. As $\operatorname{Ad}(\exp \bar{X})=\sum_{m=0}^{\infty}(1 / m !)(\operatorname{ad} \bar{X})^{m}$, we have

$$
\operatorname{Id}_{\mathfrak{u}}-\left.\operatorname{ad} \bar{X}\right|_{\mathfrak{u}}+\bar{D}_{\bar{X}}=\left.\operatorname{Ad}\left(\bar{u}^{-1}\right)\right|_{\mathfrak{u}}=\left.\operatorname{Ad}\left(b^{-1}\right) \operatorname{Ad}(z u)^{-1}\right|_{\mathfrak{u}}=\left.\lambda^{-1} \operatorname{Ad}\left(b^{-1}\right)\right|_{\mathfrak{u}} .
$$

It follows that $\max \left(1,\left\|\bar{D}_{\bar{X}}\right\|\right) \leq|\lambda|^{-1}\|b\|$, and therefore by Corollary 29 (applied to $\bar{P}$ ) that $\max (1,\|\bar{X}\|)^{2} \ll|\lambda|^{-1}\|b\|$, or equivalently,

$$
|\lambda|\|b\| \max (1,\|\bar{X}\|) \ll\|b\|^{2} \max (1,\|\bar{X}\|)^{-1} .
$$

We can write (5) in the form

$$
\lambda \operatorname{Ad}(b) \circ \bar{D}_{\bar{X}}=\left.\left(\operatorname{Id}_{\mathfrak{g}}-\Delta\right)\right|_{\mathfrak{u}},
$$

where $\Delta=\lambda \operatorname{Ad}(b) \circ(\operatorname{Id}-\operatorname{ad} \bar{X}) \in \operatorname{End}(\mathfrak{g})$. Suppose that $\|\bar{X}\| \gg\|b\|^{2}$. Then $\|\Delta\| \ll$ $|\lambda|\|b\| \max (1,\|\bar{X}\|)<1$, and therefore $\mathrm{Id}-\Delta$ is invertible and $\left\|(\operatorname{Id}-\Delta)^{-1}\right\|=1$. It follows that $\bar{D}_{\bar{X}}$ is invertible, and therefore by Lemma 30 we infer that $\boldsymbol{P}^{w_{0}}=\overline{\boldsymbol{P}}$. Moreover, $\bar{D}_{\bar{X}}^{-1}=\left.\lambda(\operatorname{Id}-\Delta)^{-1} \circ \operatorname{Ad}(b)\right|_{\overline{\mathfrak{u}}}$, and therefore $\left\|\bar{D}_{\bar{X}}^{-1}\right\| \leq|\lambda|\|b\|$. By Lemma 31, we get $\bar{u} \in P w_{0} U$ and the $U$-part in the Bruhat decomposition of $\bar{u}$ is $\exp Y$ for $Y=\bar{D}_{\bar{X}}^{-1}(\bar{X})$. Hence $\|Y\| \leq|\lambda|\|b\|\|\bar{X}\| \ll\|\bar{X}\|^{-1}\|b\|^{2}$. This immediately implies Proposition 16. 


\section{References}

[Arthur 1989] J. Arthur, "Intertwining operators and residues, I: Weighted characters", J. Funct. Anal. 84:1 (1989), 19-84. MR 90j:22018 Zbl 0679.22011

[Bernstein 1974] I. N. Bernstein, "All reductive p-adic groups are tame”, Funk. Anal. i Priložen. 8:2 (1974), 3-6. In Russian; translated in Funct. Anal. Appl. 8:2 (1974), 91-93. MR 50 \#543

[Bernstein and Zelevinskii 1976] I. N. Bernstein and A. V. Zelevinskii, "Representations of the group $G L(n, F)$, where $F$ is a local non-Archimedean field", Uspekhi Mat. Nauk 31:3 (1976), 5-70. In Russian; translated in Russian Math. Surveys 31:3 (1976), 1-68. MR 54 \#12988 Zbl 0342.43017

[Bushnell 1990] C. J. Bushnell, “Induced representations of locally profinite groups", J. Algebra 134:1 (1990), 104-114. MR 92d:22025 Zbl 0727.22004

[Bushnell 2001] C. J. Bushnell, "Representations of reductive $p$-adic groups: localization of Hecke algebras and applications", J. London Math. Soc. (2) 63:2 (2001), 364-386. MR 2001m:22034 Zbl 1017.22011

[Bushnell and Kutzko 1993a] C. J. Bushnell and P. C. Kutzko, The admissible dual of GL( $N)$ via compact open subgroups, Annals of Mathematics Studies 129, Princeton University Press, 1993. MR 94h:22007 Zbl 0787.22016

[Bushnell and Kutzko 1993b] C. J. Bushnell and P. C. Kutzko, "The admissible dual of SL(N), I", Ann. Sci. École Norm. Sup. (4) 26:2 (1993), 261-280. MR 94a:22033 Zbl 0787.22017

[Carayol 1984] H. Carayol, "Représentations cuspidales du groupe linéaire", Ann. Sci. École Norm. Sup. (4) 17:2 (1984), 191-225. MR 86f:22019 Zbl 0549.22009

[Chevalley 1951] C. Chevalley, "Sur le groupe exceptionnel (E6)", C. R. Acad. Sci. Paris 232 (1951), 1991-1993. MR 12,802d Zbl 0043.26005

[Finis et al. 2012] T. Finis, E. Lapid, and W. Müller, "Limit multiplicities for principal congruence subgroups of GL(n)", preprint, 2012. arXiv 1208.2257

[Igusa 1970] J.-i. Igusa, "A classification of spinors up to dimension twelve", Amer. J. Math. 92 (1970), 997-1028. MR 43 \#3291 Zbl 0217.36203

[Jacquet 1971] H. Jacquet, "Représentations des groupes linéaires $p$-adiques", pp. 119-220 in Theory of group representations and Fourier analysis (Montecatini Terme, 1970), edited by F. Gherardelli, Edizioni Cremonese, Rome, 1971. MR 45 \#453 Zbl 0242.22016

[Kac 1980] V. G. Kac, "Some remarks on nilpotent orbits", J. Algebra 64:1 (1980), 190-213. MR 81i:17005 Zbl 0431.17007

[Kim 2007] J.-L. Kim, "Supercuspidal representations: an exhaustion theorem", J. Amer. Math. Soc. 20:2 (2007), 273-320. MR 2008c:22014 Zbl 1111.22015

[Lapid 2004] E. M. Lapid, "Appendix to "Absolute convergence of the spectral side of the Arthur trace formula for GL", by W. Müller and B. Speh", Geom. Funct. Anal. 14:1 (2004), 85-90. MR 2005m:22021 Zbl 1083.11031

[Lubotzky 1995] A. Lubotzky, "Subgroup growth and congruence subgroups", Invent. Math. 119:2 (1995), 267-295. MR 95m:20054 Zbl 0848.20036

[Richardson et al. 1992] R. Richardson, G. Röhrle, and R. Steinberg, "Parabolic subgroups with abelian unipotent radical”, Invent. Math. 110:3 (1992), 649-671. MR 93j:20092 Zbl 0786.20029

[Schneider and Stuhler 1997] P. Schneider and U. Stuhler, "Representation theory and sheaves on the Bruhat-Tits building”, Inst. Hautes Études Sci. Publ. Math. 85 (1997), 97-191. MR 98m:22023 Zbl 0892.22012

[Serre 2001] J.-P. Serre, Complex semisimple Lie algebras, Springer, Berlin, 2001. MR 2001h:17001 Zbl 1058.17005 
[Shahidi 1981] F. Shahidi, "On certain L-functions", Amer. J. Math. 103:2 (1981), 297-355. MR 82i: 10030 Zbl 0467.12013

[Silberger 1979] A. J. Silberger, Introduction to harmonic analysis on reductive p-adic groups, Mathematical Notes 23, Princeton University Press, 1979. MR 81m:22025 Zbl 0458.22006

[Stevens 2008] S. Stevens, "The supercuspidal representations of $p$-adic classical groups", Invent. Math. 172:2 (2008), 289-352. MR 2010e:22008 Zbl 1140.22016

[Tits 1979] J. Tits, "Reductive groups over local fields", pp. 29-69 in Automorphic forms, representations and L-functions, Part 1 (Corvallis, OR, 1977), edited by A. Borel and W. Casselman, Proc. Sympos. Pure Math. 33, Amer. Math. Soc., Providence, RI, 1979. MR 80h:20064 Zbl 0415.20035

[Waldspurger 2003] J.-L. Waldspurger, "La formule de Plancherel pour les groupes $p$-adiques (d'après Harish-Chandra)", J. Inst. Math. Jussieu 2:2 (2003), 235-333. MR 2004d:22009 Zbl 1029.22016

[Yu 2001] J.-K. Yu, "Construction of tame supercuspidal representations", J. Amer. Math. Soc. 14:3 (2001), 579-622. MR 2002f:22033 Zbl 0971.22012

Received June 11, 2012.

TOBIAS FINIS

INSTITUT FÜR MATHEMATIK

FREIE UNIVERSITÄT BERLIN

ARNimAlLEe 3

D-14195 BERLIN

GERMANY

finis@math.fu-berlin.de

EREZ LAPID

EINSTEIN Institute of MATHEMATICS

The Hebrew University of Jerus ALEM

91904 JERUSALEM

ISRAEL

erezla@math.huji.ac.il

WERNER MÜLLER

MATHEMATISCHES INSTITUT

RHEINISCHE FRIEDRICH-WILHELMS-UNIVERSITÄT BONN

ENDENICHER ALLEE 60

D-53115 BONN

GERMANY

mueller@math.uni-bonn.de 


\title{
PACIFIC JOURNAL OF MATHEMATICS
}

\author{
http://pacificmath.org
}

Founded in 1951 by E. F. Beckenbach (1906-1982) and F. Wolf (1904-1989)

\section{EDITORS}

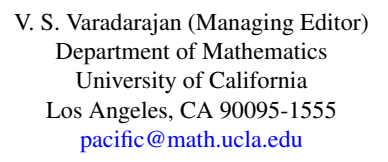

Don Blasius

Department of Mathematics University of California

Los Angeles, CA 90095-1555

blasius@math.ucla.edu

Robert Finn

Department of Mathematics

Stanford University

Stanford, CA 94305-2125

finn@math.stanford.edu

Alexander Merkurjev

Department of Mathematics

University of California

Los Angeles, CA 90095-1555

merkurev@math.ucla.edu

\author{
Vyjayanthi Chari \\ Department of Mathematics \\ University of California \\ Riverside, CA 92521-0135 \\ chari@math.ucr.edu \\ Kefeng Liu \\ Department of Mathematics \\ University of California \\ Los Angeles, CA 90095-1555 \\ liu@math.ucla.edu \\ Sorin Popa \\ Department of Mathematics \\ University of California \\ Los Angeles, CA 90095-1555 \\ popa@math.ucla.edu \\ Paul Yang \\ Department of Mathematics \\ Princeton University \\ Princeton NJ 08544-1000 \\ yang@math.princeton.edu
}

\section{PRODUCTION}

Silvio Levy, Scientific Editor, pacific@math.berkeley.edu

\section{SUPPORTING INSTITUTIONS}

ACADEMIA SINICA, TAIPEI

CALIFORNIA INST. OF TECHNOLOGY

INST. DE MATEMÁTICA PURA E APLICADA

KEIO UNIVERSITY

MATH. SCIENCES RESEARCH INSTITUTE

NEW MEXICO STATE UNIV.

OREGON STATE UNIV.

\author{
STANFORD UNIVERSITY \\ UNIV. OF BRITISH COLUMBIA \\ UNIV. OF CALIFORNIA, BERKELEY \\ UNIV. OF CALIFORNIA, DAVIS \\ UNIV. OF CALIFORNIA, LOS ANGELES \\ UNIV. OF CALIFORNIA, RIVERSIDE \\ UNIV. OF CALIFORNIA, SAN DIEGO \\ UNIV. OF CALIF., SANTA BARBARA
}

\author{
Daryl Cooper \\ Department of Mathematics \\ University of California \\ Santa Barbara, CA 93106-3080 \\ cooper@math.ucsb.edu \\ Jiang-Hua Lu \\ Department of Mathematics \\ The University of Hong Kong \\ Pokfulam Rd., Hong Kong \\ jhlu@maths.hku.hk
}

\section{Jie Qing}

Department of Mathematics

University of California

Santa Cruz, CA 95064

qing@cats.ucsc.edu

These supporting institutions contribute to the cost of publication of this Journal, but they are not owners or publishers and have no responsibility for its contents or policies.

See inside back cover or pacificmath.org for submission instructions.

The subscription price for 2012 is US \$420/year for the electronic version, and \$485/year for print and electronic.

Subscriptions, requests for back issues from the last three years and changes of subscribers address should be sent to Pacific Journal of Mathematics, P.O. Box 4163, Berkeley, CA 94704-0163, U.S.A. Prior back issues are obtainable from Periodicals Service Company, 11 Main Street, Germantown, NY 12526-5635. The Pacific Journal of Mathematics is indexed by Mathematical Reviews, Zentralblatt MATH, PASCAL CNRS Index, Referativnyi Zhurnal, Current Mathematical Publications and the Science Citation Index.

The Pacific Journal of Mathematics (ISSN 0030-8730) at the University of California, c/o Department of Mathematics, 969 Evans Hall, Berkeley, CA 94720-3840, is published monthly except July and August. Periodical rate postage paid at Berkeley, CA 94704, and additional mailing offices. POSTMASTER: send address changes to Pacific Journal of Mathematics, P.O. Box 4163, Berkeley, CA 94704-0163.

PJM peer review and production are managed by EditFlow ${ }^{\circledR}$ from Mathematical Sciences Publishers.

\section{PUBLISHED BY}

\section{mathematical sciences publishers}

http://msp.org/

A NON-PROFIT CORPORATION

Typeset in LATEX 


\section{PACIFIC JOURNAL OF MATHEMATICS}

Volume $260 \quad$ No. $2 \quad$ December 2012

\section{Special issue \\ devoted to the memory of Jonathan Rogawski}

In memoriam: Jonathan Rogawski

257

DON BLASIUS, DINAKAR RAMAKRISHNAN and V. S. VARADARAJAN

$p$-adic Rankin $L$-series and rational points on CM elliptic curves

261

Massimo Bertolini, Henri DARMON and KARTIK PRASANNA

The syntomic regulator for $K_{4}$ of curves

AMNON BESSER and ROB DE JEU

Unique functionals and representations of Hecke algebras

381

BENJAMIN BRUBAKER, DANIEL BUMP and SOLOMON FRIEDBERG

A relative trace formula for PGL(2) in the local setting

395

BROOKE FEIGON

On the degrees of matrix coefficients of intertwining operators

433

TOBIAS FINIS, EREZ LAPID and WERNER MÜLlER

Comparison of compact induction with parabolic induction

457

Guy HENNIART and MARIE-FranCE Vigneras

The functional equation and beyond endoscopy

497

P. EDWARD HERMAN

A correction to Conducteur des Représentations du groupe linéaire

HERVÉ JACQUET

Modular $L$-values of cubic level

ANDREW KNIGHTLY and CHARLES LI

On occult period maps

STEPHEN KUDLA and MiCHAEL RAPOPORT

A prologue to "Functoriality and reciprocity", part I

ROBERT LANGLANDS

Truncation of Eisenstein series

EREZ LAPID and KeITH OUELLETTE

Some comments on Weyl's complete reducibility theorem

JONATHAN ROGAWSKI and V. S. VARADARAJAN

On equality of arithmetic and analytic factors through local Langlands correspondence

FREYDOON SHAHIDI 